\title{
Non-supersymmetric asymmetric orbifolds with vanishing cosmological constant
}

\author{
Yuji Satoh, ${ }^{a}$ Yuji Sugawara $^{b}$ and Taiki Wada ${ }^{b}$ \\ ${ }^{a}$ Institute of Physics, University of Tsukuba, \\ Ibaraki 305-8571, Japan \\ ${ }^{b}$ Department of Physical Sciences, College of Science and Engineering, Ritsumeikan University, \\ Shiga 525-8577, Japan \\ E-mail: ysatoh@het.ph.tsukuba.ac.jp, ysugawa@se.ritsumei.ac.jp, \\ rp0017xp@ed.ritsumei.ac.jp
}

ABSTRACT: We study type II string vacua defined by torus compactifications accompanied by T-duality twists. We realize the string vacua, specifically, by means of the asymmetric orbifolding associated to the chiral reflections combined with a shift, which are interpreted as describing the compactification on 'T-folds'. We discuss possible consistent actions of the chiral reflection on the Ramond-sector of the world-sheet fermions, and explicitly construct non-supersymmetric as well as supersymmetric vacua. Above all, we demonstrate a simple realization of non-supersymmetric vacua with vanishing cosmological constant at one loop. Our orbifold group is generated only by a single element, which results in simpler models than those with such property known previously.

Keywords: Conformal Field Models in String Theory, Superstring Vacua

ARXiv EPrint: 1512.05155 


\section{Contents}

1 Introduction 1

2 Preliminaries: building blocks for asymmetric orbifolds 3

2.1 Bosonic $T_{\text {fiber }}^{4}$ sector 3

2.2 Fermionic sector 6

3 String vacua on T-folds $\quad 8$

$\begin{array}{llr}3.1 & \text { Supersymmetric vacua } & 9\end{array}$

3.2 Non-SUSY string vacua with vanishing cosmological constant $\quad 10$

$\begin{array}{lll}3.3 & \text { Asymmetric/generalized orbifolds and T-folds } & 11\end{array}$

4 Analysis on spectra $\quad 12$

4.1 Massless spectra in the untwisted sectors 12

$\begin{array}{lll}4.2 \text { Unitarity } & 14\end{array}$

$\begin{array}{ll}4.3 & \text { Absence of winding tachyons } \\ \end{array}$

$\begin{array}{llr}5 & \text { Summary and discussions } & 18\end{array}$

$\begin{array}{ll}\text { A Summary of conventions and useful formulas } & 19\end{array}$

$\begin{array}{ll}\text { B Summary of building blocks } & 20\end{array}$

\section{Introduction}

Compactifications on non-geometric backgrounds have been receiving increasing attention in superstring theory. A particularly interesting class of such backgrounds is formulated as the fibrations of which the transition functions involve the duality transformations in string theory [1-3]. For T-duality, for instance, one then has 'T-folds' [4]. Another interesting class is the backgrounds with non-geometric fluxes that do not have naive geometrical origins in higher dimensional theories. In some cases, these are reduced to geometric ones by dualities, but are truly non-geometric in general [5-7].

These string vacua on non-geometric backgrounds are described by the world-sheet conformal field theory (CFT) on the same footing as geometric ones. We should emphasize that many of such vacua are well-defined only at particular points on the moduli space, at which enhanced symmetries emerge and the $\alpha^{\prime}$-corrections become important. The worldsheet CFT approach would provide reliable descriptions of strings even in such backgrounds. In this respect, a simple and important class of non-geometric backgrounds is realized as asymmetric orbifolds [8], in which the left- and the right-movers of strings propagate on different geometries. Especially, as typical T-duality twists are identified with chiral 
reflections, simple examples of T-folds are realized as the orbifolds by the chiral reflection combined with the shift in the base circle. These types of string vacua have been studied based on the world-sheet CFT e.g. in [9-16]. ${ }^{1}$

In this paper, we study type II string vacua defined by torus compactifications twisted by T-duality transformations in the above sense. We carefully discuss possible consistent actions of the chiral reflection on the Ramond sector of the world-sheet fermions, and explicitly construct non-supersymmetric as well as supersymmetric (SUSY) vacua. ${ }^{2}$ Among others, we present a simple realization of non-SUSY vacua with vanishing cosmological constant at the one-loop level, at least. Namely, we construct the string vacua realizing the bose-fermi cancellation despite the absence of any supercharges in space-time. Previous constructions of such string vacua are found e.g. in [21-27]. ${ }^{3}$ A novel feature, as well as an advantage, in our construction is that we only have to utilize a cyclic orbifold, in which the orbifold group is generated by a single element, and hence the construction looks rather simpler than previous ones given in those papers. It would be notable that one can achieve (nearly) vanishing cosmological constant without SUSY in a fairly simple way in the framework of non-geometric string compactifications. Our construction suggests that they would provide useful grounds also for the cosmological constant problem.

To be more precise, we first analyze in some detail the asymmetric orbifolds representing T-folds, where the partition sums from each sector in the total partition function are combined according to the windings around the 'base' circle. It turns out that the consistent action of the chiral reflections therein is not unique, from which a variety of supersymmetric T-fold vacua can be derived. As general for asymmetric orbifolds, the moduli of the internal ('fiber') tori are fixed for consistency, while a continuous radius of the base circle remains. The supersymmetry is broken by further implementing the Scherk-Schwarz type boundary condition for the world-sheet fermions [33, 34] along the base circle. In the case where the chiral reflections act as $\mathbb{Z}_{4}$ transformations in a fermionic sector, the resultant world-sheet torus partition function and hence the one-loop cosmological constant vanish: if the partition sum for the left-moving fermions is non-vanishing in a winding sector, that for the right-moving fermions vanishes, and vice versa. It is crucial here that the chiral partition sums for the fermions depend on the winding numbers in an asymmetric way. We see that all the ingredients in our setup, i.e., T-folds (asymmetric orbifolds, base winding), careful treatment of the chiral reflections and the Scherk-Schwarz twist, cooperate in this mechanism. Although we focus on specific examples in this paper, our construction would be more general. It provides a systematic way to find string vacua of T-folds, and a novel mechanism for non-supersymmetric string vacua with vanishing cosmological constant at one-loop.

This paper is organized as follows: In section 2, which is a preliminary section, we survey the building blocks (partition sums) for the modular invariant partition functions of the asymmetric orbifolds discussed later, specifying how to achieve the modular covariance

\footnotetext{
${ }^{1}$ For the aspects of non-commutativity in non-geometric backgrounds, see e.g. [13, 17-20].

${ }^{2}$ For non-supersymmetric orbifolds in heterotic string theory, see e.g. [29-32] and references therein.

${ }^{3}$ In the papers [21-23], the authors further conjectured that the cosmological constant remains vanishing at two and higher loops. However, a careful world-sheet analysis [28] shows that it does not actually vanish at two loops in those models, at least pointwise on the moduli space.
} 
in relevant sectors. Though this part might be slightly technical, the results, especially those for the fermionic sector presented in subsection 2.2, are important in the later discussion both on T-fold vacua and on vanishing cosmological constant. The readers may refer only to the definitions of the building blocks, if they are interested mostly in the physical consequences.

In section 3, we begin our main analysis of type II string vacua compactified on asymmetric orbifolds/T-folds. We first consider the supersymmetric ones. The SUSY breaking is then discussed by further incorporating the Scherk-Schwarz twist, which leads us to the non-SUSY vacua implementing the bose-fermi cancellation. In section 4 , we analyze the spectra of the physical states and check the unitarity, mainly focusing on the case of the non-SUSY vacua. We also demonstrate the absence of the instability caused by the winding tachyons, which would be typically possible for the Scherk-Schwarz compactification. We conclude with a summary and a discussion for possible future directions in section 5 .

\section{Preliminaries: building blocks for asymmetric orbifolds}

In this paper, we would like to study the type II string vacua constructed from asymmetric orbifolds of the 10-dimensional flat background given by

$$
M^{4} \times S^{1} \times \mathbb{R}_{\text {base }} \times T_{\text {fiber }}^{4}
$$

where $M^{4}\left(X^{0,1,2,3}\right.$-directions) is the 4-dimensional Minkowski space-time. Intending the twisted compactification of the 'base space' $\mathbb{R}_{\text {base }}$ ( $X^{5}$-direction), we consider the orbifolding defined by the twist operator $\mathcal{T}_{2 \pi R} \otimes \sigma: \mathcal{T}_{2 \pi R}$ is the translation along the base direction by $2 \pi R$, and $\sigma$ denotes an automorphism acting on the 'fiber sector' $T_{\text {fiber }}^{4}\left(X^{6,7,8,9}\right)$, which is specified in detail later. We especially focus on the cases where $\sigma$ acts as the 'chiral reflection', or the T-duality transformation,

$$
\sigma:\left(X_{L}^{i}, X_{R}^{i}\right) \longmapsto\left(X_{L}^{i},-X_{R}^{i}\right), \quad(i=6,7,8,9) .
$$

The $S^{1}$-factor ( $X^{4}$-direction) in $(2.1)$ is not important in our arguments. We begin our analysis by specifying the relevant bosonic and fermionic sectors and their chiral blocks that compose the modular invariants for our asymmetric orbifolds.

\subsection{Bosonic $T_{\text {fiber }}^{4}$ sector}

In the bosonic sector, let us first consider the 4-dimensional torus with the $\mathrm{SO}(8)$-symmetry enhancement which we denote as $T^{4}[\mathrm{SO}(8)]$, in order that the relevant asymmetric orbifold action (chiral reflection) is well-defined. The torus partition function of this system is

$$
Z_{T^{4}[\mathrm{SO}(8)]}(\tau, \bar{\tau})=\frac{1}{2}\left\{\left|\frac{\theta_{3}}{\eta}\right|^{8}+\left|\frac{\theta_{4}}{\eta}\right|^{8}+\left|\frac{\theta_{2}}{\eta}\right|^{8}\right\} .
$$

Another system that is compatible with our asymmetric orbifolding and of our interests is the product of the 2-dimensional tori with the $\mathrm{SO}(4)$-symmetry, $T^{2}[\mathrm{SO}(4)] \times T^{2}[\mathrm{SO}(4)]$, 
the partition function of which is given by

$$
Z_{\left.T^{2}[\mathrm{SO}(4)] \times T^{2}[\mathrm{SO}(4)]\right]}(\tau, \bar{\tau})=\frac{1}{4}\left\{\left|\frac{\theta_{3}}{\eta}\right|^{4}+\left|\frac{\theta_{4}}{\eta}\right|^{4}+\left|\frac{\theta_{2}}{\eta}\right|^{4}\right\}^{2}
$$

It is useful to note the equivalence

$$
T^{2}[\mathrm{SO}(4)] \times T^{2}[\mathrm{SO}(4)] \cong T^{4}[\mathrm{SO}(8)] / \mathbb{Z}_{2} \cong\left[S^{1}[\mathrm{SU}(2)]\right]^{4},
$$

where $S^{1}[\mathrm{SU}(2)]$ expresses the circle of the self-dual radius $R=1 .{ }^{4}$ Namely, while both of $X^{6,7}$ and $X^{8,9}$ are compactified on the 2 -torus $T^{2}[\mathrm{SO}(4)]$ at the fermionic point with radius $\sqrt{2}$, the following four compact bosons have the self-dual radius,

$$
Y_{ \pm}^{1}:=\frac{1}{\sqrt{2}}\left(X^{6} \pm i X^{7}\right), \quad Y_{ \pm}^{2}:=\frac{1}{\sqrt{2}}\left(X^{8} \pm i X^{9}\right) .
$$

The equivalence (2.5) is confirmed by the simple identities (B.3).

We then consider the action of the automorphism $\sigma$ for $T^{4}[\mathrm{SO}(8)]$ and $T^{2}[\mathrm{SO}(4)] \times$ $T^{2}[\mathrm{SO}(4)]$. Since relative phases for the left and the right movers are generally possible in asymmetric orbifolding, in addition to the action without phases, we consider an action with phases according to [10] for $T^{2}[\mathrm{SO}(4)] \times T^{2}[\mathrm{SO}(4)]$. In total, we consider the following three cases as models relevant to our construction of string vacua given in section 3 . This means that the moduli of $T_{\text {fiber }}^{4}$ need be restricted to the particular points given here, while the radius of $S_{\text {base }}^{1}$ can be freely chosen. We particularly elaborate on the derivation of the building blocks for the case of $T^{4}[\mathrm{SO}(8)]$, and mention on other cases briefly. The explicit forms of the relevant building blocks are summarized in appendix B. The case with phases for $T^{4}[\mathrm{SO}(8)]$ can be similarly discussed following $[10,16]$, although we do not work on it in this paper.

1. Chiral reflection in $\boldsymbol{T}^{4}[\mathbf{S O}(8)]$. We start with $T^{4}[\mathrm{SO}(8)]$. In this case, the orbifold action is defined by the chiral reflection (2.2) acting only on the right-moving components. We simply assume $\sigma$ acts as the identity operator on any states in the left-mover, and also that $\sigma^{2}$ acts as the identity over the Hilbert space of the untwisted sector of the orbifolds of our interest. ${ }^{5}$ We note that the action of $\sigma^{2}$ on the twisted sectors should be determined so that it preserves the modular invariance of the total system, and does not necessarily coincide with the identity. This is a characteristic feature of asymmetric orbifolds. See for example [35].

Let us evaluate the building blocks in this sector of the torus partition function. These are schematically written as

$$
F_{(a, b)}^{T^{4}[\mathrm{SO}(8)]}(\tau, \bar{\tau}):=\operatorname{Tr}_{\sigma^{a} \text {-twisted sector }}\left[\sigma^{b} q^{L_{0}-\frac{c}{24}} \bar{q}^{\tilde{L}_{0}-\frac{c}{24}}\right] .
$$

\footnotetext{
${ }^{4}$ Throughout this paper, we use the $\alpha^{\prime}=1$ convention.

${ }^{5}$ This assumption is not necessarily obvious. Actually, if we fermionize the string coordinates along $T^{4}[\mathrm{SO}(8)]$, we can also realize more general situations as in our discussion given in subsection 2.2. We do not study these cases for simplicity in this paper.
} 
Here, we allow $a, b$ to be any integers despite a periodicity, which is at most of order 4 as seen below, since we later identify them as the winding numbers along the base circle $S_{\text {base }}^{1}$. We can most easily determine the building blocks $F_{(a, b)}^{T^{4}[\mathrm{SO}(8)]}$ by requiring the modular covariance,

$$
\begin{aligned}
& \left.F_{(a, b)}^{T^{4}[\mathrm{SO}(8)]}(\tau, \bar{\tau})\right|_{S}=F_{(b,-a)}^{T^{4}[\mathrm{SO}(8)]}(\tau, \bar{\tau}), \\
& \left.F_{(a, b)}^{T^{4}[\mathrm{SO}(8)]}(\tau, \bar{\tau})\right|_{T}=F_{(a, a+b)}^{T^{4}[\mathrm{SO}(8)]}(\tau, \bar{\tau}),
\end{aligned}
$$

together with the trace over the untwisted sector,

$$
F_{(0, b)}^{T^{4}[\mathrm{SO}(8)]}(\tau, \bar{\tau})=\overline{\left(\frac{\theta_{3} \theta_{4}}{\eta^{2}}\right)^{2}} \cdot \frac{1}{2}\left\{\left(\frac{\theta_{3}}{\eta}\right)^{4}+\left(\frac{\theta_{4}}{\eta}\right)^{4}\right\}, \quad\left({ }^{\forall} b \in 2 \mathbb{Z}+1\right) .
$$

Then, the desired building blocks are found to be

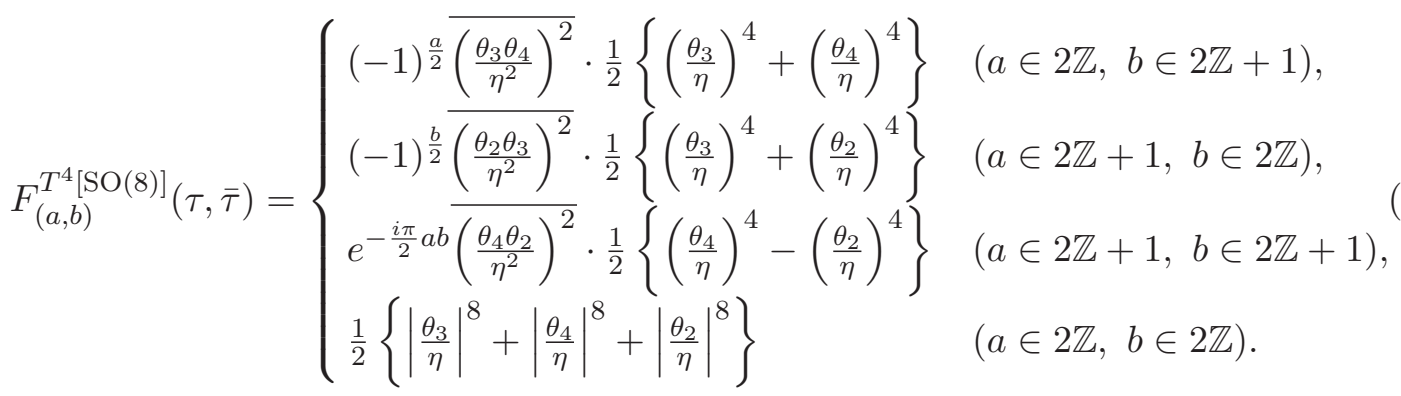

One can confirm that they indeed satisfy the modular covariance relations (2.8).

2. Chiral reflection in $\boldsymbol{T}^{2}[\mathrm{SO}(4)] \times \boldsymbol{T}^{2}[\mathrm{SO}(4)]$. In the first case of $T^{2}[\mathrm{SO}(4)] \times$ $T^{2}[\mathrm{SO}(4)]$ or the $\mathbb{Z}_{2}$-orbifold of $T^{4}[\mathrm{SO}(8)]$, we may consider the same orbifold action $\sigma$ as given in case 1, Namely, it acts as the identity on the left-mover, and assumes $\sigma^{2}=1$ in the untwisted sector. The modular covariant building blocks of the torus partition function are just determined in the same way as above. We present them in (B.2) in appendix B.

3. Chiral reflection in $T^{2}[\mathrm{SO}(4)] \times T^{2}[\mathrm{SO}(4)]$ with a phase factor. In the second case of $T^{2}[\mathrm{SO}(4)] \times T^{2}[\mathrm{SO}(4)]$, we include the phase factors for the Fock vacua when defining $\sigma$, while the action of the chiral reflection (2.2) is kept unchanged. To be more specific, recalling the equivalence (2.5), let us introduce 4 copies of the $\mathrm{SU}(2)$-current algebra of level 1 whose third components are identified as

$$
J^{3,(i)}=i \partial Y_{+}^{1}, i \partial Y_{-}^{1}, i \partial Y_{+}^{2}, i \partial Y_{-}^{2}, \quad(i=1, \ldots, 4),
$$

where $Y$ 's are the compact bosons in (2.6). With these currents, $\sigma$ is now explicitly defined according to [10] by

$$
\sigma:=\prod_{i=1}^{4}\left[e^{i \pi J_{L, 0}^{3,(i)}} \otimes e^{i \pi J_{R, 0}^{1,(i)}}\right] .
$$

We then obtain the building blocks according to the same procedure: the blocks for the $(0, b)$-sectors with ${ }^{\forall} b \in 2 \mathbb{Z}+1$ are computed first, and then those for other sectors are 
obtained by requiring the modular covariance. It turns out that these are eventually equal to the building blocks of the symmetric $\mathbb{Z}_{2}$-orbifold defined by

$$
\left(X_{L}^{i}, X_{R}^{i}\right) \longmapsto\left(-X_{L}^{i},-X_{R}^{i}\right), \quad\left({ }^{\forall} i=6,7,8,9\right) .
$$

Of course, this fact is not surprising since (2.12) is equivalent to the symmetric one

$$
\prod_{i=1}^{4}\left[e^{i \pi J_{L, 0}^{1,(i)}} \otimes e^{i \pi J_{R, 0}^{1,(i)}}\right]
$$

by an automorphism of $\mathrm{SU}(2)^{\otimes 4}$, as was pointed out in [11]. We exhibit the building blocks in this case in (B.4).

\subsection{Fermionic sector}

We next consider the fermionic sector. The orbifold action should act on the world-sheet fermions as

$$
\sigma:\left(\psi_{L}^{i}, \psi_{R}^{i}\right) \longmapsto\left(\psi_{L}^{i},-\psi_{R}^{i}\right), \quad(i=6,7,8,9),
$$

to preserve the world-sheet superconformal symmetry. (2.15) uniquely determines the action on the Hilbert space of the NS-sector. However, it is not on the R-sector, and as is discussed in the next section, we obtain different string vacua according to its choice. The fermionic part is thus crucial in our analysis. In the following, we include the fermions $\psi^{i}$ $(i=2,3,4,5)$ in other transverse part from $M^{4} \times S^{1} \times S_{\text {base }}^{1}$, on which $\sigma$ acts trivially. If retaining the Poincare symmetry in 4 dimensions, we then have two possibilities, which can be understood from the point of view of bosonization as follows:

(i) $\mathbb{Z}_{\mathbf{2}}$ action on the untwisted R-sector. In this case, we bosonize $\psi_{R}^{i}(i=2, \ldots, 9)$ as

$$
\begin{aligned}
\psi_{R}^{2} \pm i \psi_{R}^{3} \equiv \sqrt{2} e^{ \pm i H_{0, R}}, & \psi_{R}^{4} \pm i \psi_{R}^{5} \equiv \sqrt{2} e^{ \pm i H_{1, R}} \\
\psi_{R}^{6} \pm i \psi_{R}^{7} \equiv \sqrt{2} e^{ \pm i H_{2, R}}, & \psi_{R}^{8} \pm i \psi_{R}^{9} \equiv \sqrt{2} e^{ \pm i H_{3, R}}
\end{aligned}
$$

and define the spin fields for $\mathrm{SO}(8)$ as

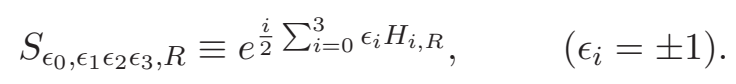

Then, (2.15) translates into

$$
\sigma:\left(H_{0, R}, H_{1, R}, H_{2, R}, H_{3, R}\right) \longmapsto\left(H_{0, R}, H_{1, R}, H_{2, R}+\pi, H_{3, R}+\pi\right),
$$

and thus, we find $\sigma^{2}=\mathbf{1}$ for all the states in the NS and R-sectors in the untwisted sector. This type of twisting preserves half of the space-time SUSY. In fact, the Ramond vacua that are generated by the spin fields (2.17) survive the $\sigma$-projection when $\epsilon_{2}+\epsilon_{3}=0$. 
(ii) $\mathbb{Z}_{\mathbf{4}}$ action on the untwisted $\mathbf{R}$-sector. In this case, we bosonize $\psi_{R}^{i}(i=2, \ldots, 9)$ as

$$
\begin{aligned}
H_{0, R}^{\prime} & \equiv H_{0, R}, & \psi_{R}^{4} \pm i \psi_{R}^{6} & \equiv \sqrt{2} e^{ \pm i H_{1, R}^{\prime}}, \\
\psi_{R}^{5} \pm i \psi_{R}^{7} & \equiv \sqrt{2} e^{ \pm i H_{2, R}^{\prime},} & H_{3, R}^{\prime} & \equiv H_{3, R},
\end{aligned}
$$

and define the spin fields for $\mathrm{SO}(8)$ as

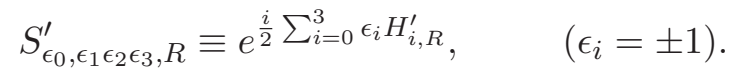

This time, (2.15) translates into

$$
\sigma:\left(H_{0, R}^{\prime}, H_{1, R}^{\prime}, H_{2, R}^{\prime}, H_{3, R}^{\prime}\right) \longmapsto\left(H_{0, R}^{\prime},-H_{1, R}^{\prime},-H_{2, R}^{\prime}, H_{3, R}^{\prime}+\pi\right) .
$$

Then, $\sigma^{2}=-\mathbf{1}$ for the R-sector, while $\sigma^{2}=\mathbf{1}$ still holds for the NS sector. In other words, we have found in this second case that

$$
\sigma^{2}=(-1)^{F_{R}},
$$

where $F_{R}$ denotes the 'space-time fermion number' (mod 2) from the right-mover. The operator $(-1)^{F_{R}}$ acts as the sign flip on all the states belonging to the right-moving R-sector.

As long as the $M^{4}$ part or $\psi^{2,3}$ are kept intact, other possibilities essentially reduce to one of these two. The chiral blocks of the right-moving fermions in the eight-dimensional transverse part are then determined in the same way as in the bosonic $T^{4}$ sector: we first evaluate the trace over the untwisted sector with the insertion of $\sigma^{b}$, and next require the modular covariance.

For case (i), we then have the desired chiral blocks $\overline{f_{(a, b)}(\tau)}$ with

$$
f_{(a, b)}(\tau)= \begin{cases}(-1)^{\frac{a}{2}}\left\{\left(\frac{\theta_{3}}{\eta}\right)^{2}\left(\frac{\theta_{4}}{\eta}\right)^{2}-\left(\frac{\theta_{4}}{\eta}\right)^{2}\left(\frac{\theta_{3}}{\eta}\right)^{2}+0\right\} & (a \in 2 \mathbb{Z}, b \in 2 \mathbb{Z}+1), \\ (-1)^{\frac{b}{2}}\left\{\left(\frac{\theta_{3}}{\eta}\right)^{2}\left(\frac{\theta_{2}}{\eta}\right)^{2}+0-\left(\frac{\theta_{2}}{\eta}\right)^{2}\left(\frac{\theta_{3}}{\eta}\right)^{2}\right\} & (a \in 2 \mathbb{Z}+1, b \in 2 \mathbb{Z}), \\ -e^{\frac{i \pi}{2} a b}\left\{0+\left(\frac{\theta_{2}}{\eta}\right)^{2}\left(\frac{\theta_{4}}{\eta}\right)^{2}-\left(\frac{\theta_{4}}{\eta}\right)^{2}\left(\frac{\theta_{2}}{\eta}\right)^{2}\right\} & (a \in 2 \mathbb{Z}+1, b \in 2 \mathbb{Z}+1), \\ \left(\frac{\theta_{3}}{\eta}\right)^{4}-\left(\frac{\theta_{4}}{\eta}\right)^{4}-\left(\frac{\theta_{2}}{\eta}\right)^{4} & (a \in 2 \mathbb{Z}, b \in 2 \mathbb{Z}) .\end{cases}
$$

Each term from the left to the right corresponds to the NS, $\widetilde{\mathrm{NS}}$, and R sector, respectively, where the ' $\widetilde{\mathrm{NS}}$ ' denotes the NS-sector with $(-1)^{f}$ inserted $(f$ is the world-sheet fermion number). These trivially vanish as expected from the space-time SUSY.

We note that in the fermionic sectors the modular covariance means: ${ }^{6}$

$$
\left.f_{a, b}(\tau)\right|_{S}=f_{(b,-a)}(\tau),\left.\quad f_{(a, b)}(\tau)\right|_{T}=-e^{-2 \pi i \frac{1}{6}} f_{(a, a+b)}(\tau),
$$

with the phase for the T-transformation. Since the total blocks for the transverse fermions consist of $\overline{f_{(a, b)}(\tau)}$ and the left-moving part,

$$
\mathcal{J}(\tau) \equiv\left(\frac{\theta_{3}}{\eta}\right)^{4}-\left(\frac{\theta_{4}}{\eta}\right)^{4}-\left(\frac{\theta_{2}}{\eta}\right)^{4},
$$

\footnotetext{
${ }^{6}$ Since $f_{(a, b)}(\tau)$ vanish, $(2.24)$ may appear to be subtle. Hence, we present a more rigid interpretation of modular covariance in appendix B.
} 
eq. (2.24) indeed assures the proper modular covariance:

$$
\left.\left[\mathcal{J}(\tau) \overline{f_{(a, b)}(\tau)}\right]\right|_{S}=\mathcal{J}(\tau) \overline{f_{(b,-a)}(\tau)},\left.\quad\left[\mathcal{J}(\tau) \overline{f_{(a, b)}(\tau)}\right]\right|_{T}=\mathcal{J}(\tau) \overline{f_{(a, a+b)}(\tau)} .
$$

We next consider the chiral blocks for case (ii), which we denote by $\overline{\mathbf{f}_{(a, b)}(\tau)}$. In this case, the treatment of the R-sector needs a little more care. First, from (2.21) we find that

$$
\mathbf{f}_{(0, b)}(\tau)=f_{(0, b)}(\tau), \quad\left({ }^{\forall} b \in 2 \mathbb{Z}+1\right),
$$

which are vanishing. On the other hand, the blocks for the sectors of $a, b \in 2 \mathbb{Z}$ are nontrivially modified due to (2.22). Again it is easy to evaluate the trace over the $(0, b)$-sector, and by requiring the modular covariance (in the sense of (2.24) or (B.8)), we finally obtain

$$
\mathbf{f}_{(a, b)}(\tau)=f_{(a, b)}(\tau), \quad(a \in 2 \mathbb{Z}+1 \text { or } b \in 2 \mathbb{Z}+1),
$$

and

$$
\mathbf{f}_{(a, b)}(\tau)= \begin{cases}\left(\frac{\theta_{3}}{\eta}\right)^{4}-\left(\frac{\theta_{4}}{\eta}\right)^{4}-\left(\frac{\theta_{2}}{\eta}\right)^{4} & (a \in 4 \mathbb{Z}, b \in 4 \mathbb{Z}), \\ \left(\frac{\theta_{3}}{\eta}\right)^{4}-\left(\frac{\theta_{4}}{\eta}\right)^{4}+\left(\frac{\theta_{2}}{\eta}\right)^{4} & (a \in 4 \mathbb{Z}, b \in 4 \mathbb{Z}+2), \\ \left(\frac{\theta_{3}}{\eta}\right)^{4}+\left(\frac{\theta_{4}}{\eta}\right)^{4}-\left(\frac{\theta_{2}}{\eta}\right)^{4} & (a \in 4 \mathbb{Z}+2, b \in 4 \mathbb{Z}), \\ -\left\{\left(\frac{\theta_{3}}{\eta}\right)^{4}+\left(\frac{\theta_{4}}{\eta}\right)^{4}+\left(\frac{\theta_{2}}{\eta}\right)^{4}\right\} & (a \in 4 \mathbb{Z}+2, b \in 4 \mathbb{Z}+2) .\end{cases}
$$

In contrast to $f_{(a, b)}$, these $\mathbf{f}_{(a, b)}$ are in general non-vanishing, which signals the SUSY breaking in the right-moving sector. This completes our construction of the chiral building blocks. These are used in the following sections.

\section{String vacua on T-folds}

Now we construct type II string vacua by combining the building blocks derived in the previous section. They are interpretable as describing the compactification on T-folds.

First, to describe the 'base sector' for $S_{\text {base }}^{1}$, we introduce the following notation,

$$
Z_{R,(w, m)}(\tau, \bar{\tau}):=\frac{R}{\sqrt{\tau_{2}}|\eta(\tau)|^{2}} e^{-\frac{\pi R^{2}}{\tau_{2}}|w \tau+m|^{2}}, \quad(w, m \in \mathbb{Z}),
$$

where $R$ is the radius of the compactification and the integers $w, m$ are identified as the spatial and temporal winding numbers. In terms of these, we find $^{7}$

$$
\operatorname{Tr}_{\text {base }}\left[\left(\mathcal{T}_{2 \pi R}\right)^{m} q^{L_{0}-\frac{c}{24}} \overline{q^{\tilde{L}_{0}-\frac{c}{24}}}\right]=Z_{R,(0, m)}(\tau, \bar{\tau}),
$$

${ }^{7}$ Here we adopt the conventional normalization of the trace for the CFT for $\mathbb{R}_{\text {base }}$,

$$
\operatorname{Tr}_{\text {base }}\left[q^{L_{0}-\frac{c}{24}} \overline{q^{\tilde{L}_{0}-\frac{c}{24}}}\right]=\frac{R}{\sqrt{\tau_{2}}|\eta|^{2}} .
$$

This means that we start with $S_{N R}^{1}$ for the base CFT with an arbitrary integer $N$, and regard the insertion of the shift operators $\left(\mathcal{T}_{2 \pi R}\right)^{m}$ as implementing the $\mathbb{Z}_{N}$-orbifolding. 
and the torus partition function of a free compact boson with radius $R$ reads

$$
Z_{R}(\tau, \bar{\tau})=\sum_{w, m \in \mathbb{Z}} Z_{R,(w, m)}(\tau, \bar{\tau}) .
$$

To calculate the total partition function, we proceed as follows: first, we evaluate

$$
\begin{aligned}
Z_{(0, m)}(\tau, \bar{\tau}) & \equiv \operatorname{Tr}_{w=0 \text { sector }}\left[\left(\mathcal{T}_{2 \pi R} \otimes \sigma\right)^{m} q^{L_{0}-\frac{c}{24}} \overline{\tilde{L}_{0}-\frac{c}{24}}\right] \\
& =Z_{R,(0, m)}(\tau, \bar{\tau}) \operatorname{Tr}_{\text {untwisted }}\left[\sigma^{m} q^{L_{0}-\frac{c}{24}} \overline{q^{\tilde{L}_{0}-\frac{c}{24}}}\right] .
\end{aligned}
$$

Second, we extend (3.4) to the partition function of the general winding sector $Z_{(w, m)}(\tau, \bar{\tau})$ by requiring the modular covariance. It is straightforward to perform this, given the relevant building blocks in the previous section. These two steps are also in parallel with the previous section. Finally, we obtain the total partition function by summing over the winding numbers $w, m \in \mathbb{Z}$ along the base circle as

$$
Z(\tau, \bar{\tau})=\sum_{w, m \in \mathbb{Z}} Z_{(w, m)}(\tau, \bar{\tau})
$$

\subsection{Supersymmetric vacua}

In this way, we can construct string vacua, depending on the combination of the bosonic $T^{4}$ sector (1-3) in section 2.1 and the transverse fermionic sector (i, ii) in section 2.2. All these are supersymmetric.

As the first example, we consider $T^{4}[\mathrm{SO}(8)]$ in the background (2.1). Choosing case (i) for the fermionic sector, we obtain the torus partition function as

$$
Z(\tau, \bar{\tau})=\frac{1}{4} Z_{M^{4} \times S^{1}}^{\mathrm{tr}}(\tau, \bar{\tau}) \sum_{w, m \in \mathbb{Z}} Z_{R,(w, m)}(\tau, \bar{\tau}) F_{(w, m)}^{T^{4}[\mathrm{SO}(8)]}(\tau, \bar{\tau}) \mathcal{J}(\tau) \overline{f_{(w, m)}(\tau)},
$$

where $Z_{M^{4} \times S^{1}}^{\mathrm{tr}}(\tau, \bar{\tau})$ denotes the bosonic partition function for the transverse part of $M^{4} \times$ $S^{1}$-sector. $\mathcal{J}(\tau)$ is the contribution from the left-moving free fermions defined in (2.25), and the overall factor $1 / 4$ is due to the chiral GSO projections. This is manifestly modular invariant by construction and defines a superstring vacuum, which preserves $3 / 4$ of the space-time SUSY, that is, 16 supercharges from the left-mover and 8 supercharges from the right-mover.

For case (ii), we replace $f_{(w, m)}(\tau)$ in $(3.6)$ with $\mathbf{f}_{(w, m)}(\tau)$ given in $(2.28),(2.29)$, and obtain the torus partition function

$$
Z(\tau, \bar{\tau})=\frac{1}{4} Z_{M^{4} \times S^{1}}^{\mathrm{tr}}(\tau, \bar{\tau}) \sum_{w, m \in \mathbb{Z}} Z_{R,(w, m)}(\tau, \bar{\tau}) F_{(w, m)}^{T^{4}[\mathrm{SO}(8)]}(\tau, \bar{\tau}) \mathcal{J}(\tau) \overline{\mathbf{f}_{(w, m)}(\tau)} .
$$

This time, we are left with the $1 / 2$ space-time SUSY that originates only from the leftmover. ${ }^{8}$

It is straightforward to construct the string vacua in other four cases based on $T^{2}[\mathrm{SO}(4)] \times T^{2}[\mathrm{SO}(4)]$ : one has only to replace the bosonic building blocks $F_{(w, m)}^{T^{4}[\mathrm{SO}(8)]}(\tau, \bar{\tau})$ in the above with (B.2) or (B.4) without any changes in other sectors.

\footnotetext{
${ }^{8}$ See the discussions given in section 3.2 for the counting of unbroken supercharges in more detail.
} 


\begin{tabular}{|c|c|c|c|c|}
\hline & base $\left(X^{5}\right)$ & $T^{4}\left(X^{6,7,8,9}\right)$ & left-moving fermions & right-moving fermions \\
\hline \hline$g^{4 n}$ & $\mathcal{T}_{2 \pi(4 n) R}$ & $\mathbf{1}$ & $\mathbf{1}$ & $\mathbf{1}$ \\
\hline$g^{4 n+1}$ & $\mathcal{T}_{2 \pi(4 n+1) R}$ & $\sigma$ & $(-1)^{F_{L}}$ & $\sigma$ \\
\hline$g^{4 n+2}$ & $\mathcal{T}_{2 \pi(4 n+2) R}$ & $\mathbf{1}$ & $\mathbf{1}$ & $(-1)^{F_{R}}$ \\
\hline$g^{4 n+3}$ & $\mathcal{T}_{2 \pi(4 n+3) R}$ & $\sigma$ & $(-1)^{F_{L}}$ & $(-1)^{F_{R}} \sigma$ \\
\hline
\end{tabular}

Table 1. Action of the twist operators $g^{n}$.

\subsection{Non-SUSY string vacua with vanishing cosmological constant}

An interesting modification of the half SUSY vacuum represented by (3.7) is to replace the base circle along the $X^{5}$-direction with the Scherk-Schwarz one [33, 34]. This means that we implement the orbifolding of the background (2.1) by the twist operator ${ }^{9}$

$$
g:=\mathcal{T}_{2 \pi R} \otimes(-1)^{F_{L}} \otimes \sigma,
$$

where $(-1)^{F_{L}}$ acts as the sign flip on any states of the left-moving Ramond sector. Again $\sigma$ denotes the chiral reflection for the $T^{4}$-sector and is assumed to satisfy $\sigma^{2}=(-1)^{F_{R}}$ as for (3.7). The action of the twist operators $g^{n}$ is summarized in table 1 .

This modification leads to the following torus partition function,

$$
Z(\tau, \bar{\tau})=\frac{1}{4} Z_{M^{4} \times S^{1}}^{\operatorname{tr}}(\tau, \bar{\tau}) \sum_{w, m \in \mathbb{Z}} Z_{R,(w, m)}(\tau, \bar{\tau}) F_{(w, m)}^{T^{4}[\mathrm{SO}(8)]}(\tau, \bar{\tau}) \mathbf{f}_{(2 w, 2 m)}(\tau) \overline{\mathbf{f}_{(w, m)}(\tau)}
$$

Here, the chiral blocks for left-moving fermions have been replaced with $\mathbf{f}_{(2 w, 2 m)}(\tau)$ as in (3.7) due to the extra twisting $(-1)^{F_{L}}$. One can confirm that this partition function vanishes for each winding sector, similarly to usual supersymmetric string vacua. Indeed, $\overline{\mathbf{f}_{(w, m)}(\tau)}=0$ for ${ }^{\forall} w \in 2 \mathbb{Z}+1$ or ${ }^{\forall} m \in 2 \mathbb{Z}+1$, while $\mathbf{f}_{(2 w, 2 m)}(\tau)=0$ for ${ }^{\forall} w, m \in 2 \mathbb{Z}$. Then, we see a bose-fermi cancellation at each mass level of the string spectrum, after making the Poisson resummation with respect to the temporal winding $m$ in a standard fashion. We will observe this aspect explicitly in section 4 . Thus, the vacuum energy or the cosmological constant in space-time vanishes at the one-loop level.

A remarkable fact here is that the space-time SUSY is nonetheless completely broken:

- For $w=0$, only the supercharges commuting with the orbifold projection $\left.\frac{1}{4} \sum_{n \in \mathbb{Z}_{4}} g^{n}\right|_{\text {fermion }}$ would be preserved. However, since the relevant projection includes both $(-1)^{F_{L}}$ and $(-1)^{F_{R}}$, all the supercharges in the unorbifolded theory cannot commute with it. This implies that all the supercharges from this sector are projected out.

${ }^{9}$ If following the notion of the original Scherk-Schwarz compactification, it would be better to introduce

$$
g^{\prime}:=\mathcal{T}_{2 \pi R} \otimes(-1)^{F^{S}} \otimes \sigma \equiv \mathcal{T}_{2 \pi R} \otimes(-1)^{F_{L}} \otimes\left[\sigma(-1)^{F_{R}}\right]
$$

instead of (3.8), where $F^{S} \equiv F_{L}+F_{R}$ is the space-time fermion number. However, the argument given here is almost unchanged even in that case, and especially, we end up with the same torus partition function (3.9). 
- For $w \neq 0$, if we had a supercharge, we would observe a bose-fermi cancellation between two sectors with winding numbers $w^{\prime}$ and $w^{\prime}+w$ for ${ }^{\forall} w^{\prime} \in \mathbb{Z}$, which would imply

$$
Z_{w^{\prime}}^{(\mathrm{NS}, \mathrm{NS})}(\tau, \bar{\tau})+Z_{w^{\prime}}^{(\mathrm{R}, \mathrm{R})}(\tau, \bar{\tau})=-\left\{Z_{w^{\prime}+w}^{(\mathrm{NS}, \mathrm{R})}(\tau, \bar{\tau})+Z_{w^{\prime}+w}^{(\mathrm{R}, \mathrm{NS})}(\tau, \bar{\tau})\right\}
$$

However, we explicitly confirm, as expected, in section 4.2 that such relations never hold for the partition function (3.9) due to the factor $Z_{R,(w, m)}(\tau, \bar{\tau})$ from the base circle.

Here, it would be worthwhile to emphasize a crucial role of the shift operator $\left.\mathcal{T}_{2 \pi R}\right|_{\text {base }}$ in the above argument. Obviously, one has a vanishing partition function even without $Z_{R,(w, m)}(\tau, \bar{\tau}):$

$$
\tilde{Z}(\tau, \bar{\tau})=\frac{1}{4 \cdot 4} Z_{M^{4} \times S^{1} \times S^{1}}^{\operatorname{tr}}(\tau, \bar{\tau}) \sum_{a, b \in \mathbb{Z}_{4}} F_{(a, b)}^{T^{4}[\mathrm{SO}(8)]}(\tau, \bar{\tau}) \mathbf{f}_{(2 a, 2 b)}(\tau) \overline{\mathbf{f}_{(a, b)}(\tau)} .
$$

For the untwisted sector with $a=0$, all the supercharges are projected out in the same way as above. However, new Ramond vacua can appear from the $a \neq 0$ sectors in this case, ${ }^{10}$ and the space-time SUSY revives eventually. The inclusion of $\left.\mathcal{T}_{2 \pi R}\right|_{\text {base }}$ was a very simple way to exclude such a possibility, since supercharges cannot carry winding charges generically, as pointed out above. This is also in accord with an intuition that in the twisted sectors masses are lifted up by the winding charges.

\subsection{Asymmetric/generalized orbifolds and T-folds}

We have explicitly constructed the non-geometric superstring vacua/partition functions, (3.6), (3.7), (3.9) for the asymmetric orbifolds associated with the chiral reflection. In this subsection, we would like to comment on the relation to the construction of Tfolds in $[10,16]$. In these works, the T-duality twists are accompanied by extra phases, so that the full operator product expansion (OPE), not only the chiral one, respects the invariance under the twist: supposed that two vertex operators including both the left and right movers are invariant, their OPE yields invariant operators. This is in accord with the ordinary principle of orbifolding by symmetries. The construction of (B.4) includes such phases and the resultant models represent the T-folds in this sense. Asymmetric orbifolding, however, generally respects the chiral OPE only, and belongs to a different class.

Here, we recall that, from the CFT point of view, T-duality is in general an isomorphism between different Hilbert spaces, which keeps the form of the Hamiltonian invariant. At the self-dual point, it acts within a single Hilbert space, but is not yet an ordinary symmetry, since the transformation to the dual fields is non-local. Thus, it may not be obvious if the OPE should fully respect the invariance under the T-duality twists. Indeed, in the case of the critical Ising model, the OPE of the order and disorder fields, which are non-local to each other, reads

$$
\sigma(z, \bar{z}) \mu(0,0) \sim|z|^{-1 / 4}\left[\omega z^{1 / 2} \psi(0)+\bar{\omega} \bar{z}^{1 / 2} \bar{\psi}(0)\right],
$$

\footnotetext{
${ }^{10}$ In fact, the orbifolding by $(-1)^{F_{L}}$ (or $(-1)^{F_{R}}$ ) acts as the 'chirality flip' of the Ramond sector, which transfers the type IIA (IIB) vacua to the type IIB (IIA) ones similarly to T-duality. See e.g. [36].
} 
where $\psi, \bar{\psi}$ are the free fermions, $\omega=\frac{1}{\sqrt{2}} e^{\frac{i \pi}{4}}$ and $\bar{\omega}$ is its complex conjugate. Under the Kramers-Wannier duality (T-duality), these fields are mapped as $(\sigma, \mu, \psi, \bar{\psi}) \rightarrow$ $(\mu, \sigma, \psi,-\bar{\psi})$. One then finds that the OPE of two invariant fields $(\sigma+\mu)(z, \bar{z})(\sigma+\mu)(0,0)$ yields non-invariant fields, since the diagonal part $\sigma \sigma+\mu \mu$ yields invariant ones.

In addition, we note that sensible CFTs may be obtained from the twists by transformations which are not the full symmetries. We refer to such CFTs as "generalized orbifold" CFTs, according to [37-39] where such CFTs are studied in the context of the topological conformal interfaces [40-43]. An application to non-geometric backgrounds has been discussed in [15]. Even though the twists are not necessarily by the full symmetries, the transformations may need to commute with the Hamiltonian, since the position of the twist operators matters otherwise. In this terminology, general asymmetric orbifold models and hence ours based on the twists without the extra phases belong to this class. In any case, our resultant models are consistent in that they are modular invariant and, as shown in the next section, have sensible spectra.

Taking these into account, we expect that the world-sheet CFTs for T-folds are generally given by the asymmetric/generalized orbifold CFTs, and that our asymmetric orbifolds without, as well as with, the extra phases also represent T-folds, as we have assumed so far (see also $[1-3,7,9,13,14]$ ). It would be an interesting issue if all these non-geometric models have the corresponding supergravity description as low-energy effective theory of T-folds. As is discussed shortly, the difference of the spectra due to the phases typically appear in the massive sector. However, the massless spectra can also differ, for example, at special points of the moduli, and thus supergravity may distinguish them.

Regarding the interpretation as T-folds, we also note that the chiral reflections both for $T^{4}[\mathrm{SO}(8)]$ and $T^{2}[\mathrm{SO}(4)] \times T^{2}[\mathrm{SO}(4)]$ are indeed realized as self-dual $O(4,4, \mathbb{Z})$ transformations which leave background geometries invariant. The elements of $O(4,4, \mathbb{Z})$ act as $\mathbb{Z}_{2}$ transformations in the untwisted bosonic sector as expected, whereas they do not generally in other sectors, for example, in the fermionic sectors (see also [10, 35]). This, however, is not a contradiction: that means that such sectors are in different representations.

\section{Analysis on spectra}

\subsection{Massless spectra in the untwisted sectors}

To clarify the physical content of the non-SUSY vacuum with the bose-fermi cancellation (3.9), let us examine the massless spectrum in the untwisted sector $(w=0)$ that survives in the low energy physics. The massless states from the twisted sectors $(w \neq 0)$ can appear only at the special radius $R$ (see subsection 4.3).

We first note the fact that all the right-moving Ramond vacua are projected out by the orbifold action $g$; recall $\sigma^{2}=(-1)^{F_{R}}$ for the world-sheet fermions. Therefore, the candidates of the bosonic and fermionic massless states only reside in the (NS, NS) and (R, NS)-sectors, respectively. It is thus enough to search the (NS, NS) and (R, NS) massless states invariant under the action of $(-1)^{F_{L}} \otimes \sigma$ within the Hilbert space of the unorbifolded theory. In this way, one can easily write down the massless spectrum. We exhibit it in 


\begin{tabular}{|c|c|c|c|c|}
\hline spin structure & left & & right & $4 \mathrm{D}$ fields \\
\hline$(\mathrm{NS}, \mathrm{NS})$ & $\begin{array}{c}\psi_{-1 / 2}^{\mu}|0\rangle \\
(\mu=2, \ldots, 9)\end{array}$ & $\otimes$ & $\begin{array}{c}\tilde{\psi}_{-1 / 2}^{\nu}|0\rangle \\
(\nu=2, \ldots, 5)\end{array}$ & $\begin{array}{l}\text { graviton, } 8 \text { vectors, } \\
14 \text { (pseudo) scalars }\end{array}$ \\
\hline$(\mathrm{R}, \mathrm{NS})$ & $\left|\epsilon_{0}, \epsilon_{1}, \epsilon_{2}, \epsilon_{3}\right\rangle$ & $\otimes$ & $\begin{array}{c}\tilde{\psi}_{-1 / 2}^{\nu}|0\rangle \\
(\nu=6, \ldots, 9)\end{array}$ & 16 Weyl fermions \\
\hline
\end{tabular}

Table 2. Massless spectrum of the non-SUSY vacuum (3.9).

\begin{tabular}{|c|ccc|c|}
\hline spin structure & left & right & 4D fields \\
\hline \hline$(\mathrm{NS}, \mathrm{NS})$ & $\psi_{-1 / 2}^{\mu}|0\rangle$ & $\otimes$ & $\tilde{\psi}_{-1 / 2}^{\nu}|0\rangle$ & graviton, 8 vectors, \\
& $(\mu=2, \ldots, 9)$ & $(\nu=2, \ldots, 5)$ & 14 (pseudo) scalars \\
\hline$(\mathrm{R}, \mathrm{R})$ & $\left|\epsilon_{0}, \epsilon_{1}, \epsilon_{2}, \epsilon_{3}\right\rangle$ & $\otimes$ & $\left|\tilde{\epsilon}_{0}, \tilde{\epsilon}_{1}, \tilde{\epsilon}_{2}, \tilde{\epsilon}_{3}\right\rangle$ & 8 vectors, \\
& & & $\left(\tilde{\epsilon}_{2}+\tilde{\epsilon}_{3}=0\right)$ & 16 (pseudo) scalars \\
\hline$(\mathrm{R}, \mathrm{NS})$ & $\left|\epsilon_{0}, \epsilon_{1}, \epsilon_{2}, \epsilon_{3}\right\rangle$ & $\otimes$ & $\tilde{\psi}_{-1 / 2}^{\nu}|0\rangle$ & 8 gravitini, \\
& & & $(\nu=2, \ldots, 5)$ & 8 Weyl fermions \\
\hline$(\mathrm{NS}, \mathrm{R})$ & $\psi_{-1 / 2}^{\mu}|0\rangle$ & $\otimes$ & $\left|\tilde{\epsilon}_{0}, \tilde{\epsilon}_{1}, \tilde{\epsilon}_{2}, \tilde{\epsilon}_{3}\right\rangle$ & 4 gravitini, \\
& $(\mu=2, \ldots, 9)$ & & $\left(\tilde{\epsilon}_{2}+\tilde{\epsilon}_{3}=0\right)$ & 12 Weyl fermions \\
\hline
\end{tabular}

Table 3. Massless spectrum of the SUSY vacuum (3.6).

\begin{tabular}{|c|ccc|c|}
\hline spin structure & left & right & 4D fields \\
\hline \hline$(\mathrm{NS}, \mathrm{NS})$ & $\psi_{-1 / 2}^{\mu}|0\rangle$ & $\otimes$ & $\tilde{\psi}_{-1 / 2}^{\mu}|0\rangle$ & graviton, 8 vectors, \\
& $(\mu=2, \ldots, 9)$ & & $(\mu=2, \ldots, 5)$ & 14 (pseudo) scalars \\
\hline$(\mathrm{R}, \mathrm{NS})$ & $\left|\epsilon_{0}, \epsilon_{1}, \epsilon_{2}, \epsilon_{3}\right\rangle$ & $\otimes$ & $\tilde{\psi}_{-1 / 2}^{\mu}|0\rangle$ & 8 gravitini, \\
& & & $(\mu=2, \ldots, 5)$ & 8 Weyl fermions \\
\hline
\end{tabular}

Table 4. Massless spectrum of the SUSY vacuum (3.7).

table 2. ${ }^{11}$ Since our background includes the $S^{1}$-factor $\left(X^{4}\right.$-direction) that is kept intact under the orbifolding, it is evident by considering T-duality that the type IIA and type IIB vacua lead us to the same massless spectra in 4 dimensions. Thus, we do not specify here which we are working on.

It is evident from table 2 that we have the same number of the massless bosonic and fermionic degrees of freedom. Nevertheless, there are no 4-dimensional gravitini, reflecting the absence of the space-time SUSY.

For comparison, it would be useful to exhibit the massless spectra in the untwisted sector for the 3/4-SUSY vacuum (3.6) and the 1/2-SUSY vacuum (3.7). We present them in table 3 and table 4 .

\footnotetext{
${ }^{11}$ Here, the '14 (pseudo) scalars' include the dilaton and the 4-dimensional axion field (dual of $B_{\mu \nu}$ ), which universally exist.
} 


\subsection{Unitarity}

The torus partition functions we constructed in the previous section include the non-trivial phase factors which originate from the requirement of the modular covariance and depend on the winding numbers along the base circle. Thus, it may not be so obvious whether the spectrum is unitary in each vacuum, though that is evident in the untwisted sector with $w=0$ by construction.

An explicit way to check the unitarity is to examine the string spectrum by the Poisson resummation of the relevant partition function with respect to the temporal winding $m$ along the base circle. To this end, we decompose the partition functions with respect to the spatial winding $w$ and the spin structures, and factor out the component of $Z_{M^{4} \times S^{1}}^{\operatorname{tr}}$ :

$$
Z(\tau, \bar{\tau})=\frac{1}{4} Z_{M^{4} \times S^{1}}^{\mathrm{tr}}(\tau, \bar{\tau}) \sum_{s, \tilde{s}} \sum_{w \in \mathbb{Z}} Z_{w}^{(s, \tilde{s})}(\tau, \bar{\tau})
$$

where $s, \tilde{s}=\mathrm{NS}, \mathrm{R}$ denote the left and right-moving spin structures.

For instance, let us pick up the non-SUSY vacuum built from $T^{4}[\mathrm{SO}(8)]$ given by (3.9). Making the Poisson resummation, we find that each function $Z_{w}^{(s, \tilde{s})}(\tau, \bar{\tau})$ with fixed $w$ becomes as follows:

- $w \in 4 \mathbb{Z}$;

$$
\begin{aligned}
Z_{w}^{(\mathrm{NS}, \mathrm{NS})}(\tau, \bar{\tau})= & -Z_{w}^{(\mathrm{R}, \mathrm{NS})}(\tau, \bar{\tau})=\frac{1}{4} \sum_{n \in \mathbb{Z}} \overline{q^{\frac{1}{4}\left(\frac{n}{2 R}-R w\right)^{2}}} q^{\frac{1}{4}\left(\frac{n}{2 R}+R w\right)^{2}} \\
& \times\left\{\left|\frac{\theta_{3}}{\eta}\right|^{8}+\left|\frac{\theta_{4}}{\eta}\right|^{8}+\left|\frac{\theta_{2}}{\eta}\right|^{8}\right\}\left|\left(\frac{\theta_{3}}{\eta}\right)^{4}-\left(\frac{\theta_{4}}{\eta}\right)^{4}\right|^{2}, \\
Z_{w}^{(\mathrm{R}, \mathrm{R})}(\tau, \bar{\tau})= & -Z_{w}^{(\mathrm{NS}, \mathrm{R})}(\tau, \bar{\tau})=\frac{1}{4} \sum_{n \in \mathbb{Z}+\frac{1}{2}} \frac{q^{\frac{1}{4}\left(\frac{n}{2 R}-R w\right)^{2}}}{q^{\frac{1}{4}\left(\frac{n}{2 R}+R w\right)^{2}}} \\
& \times\left\{\left|\frac{\theta_{3}}{\eta}\right|^{8}+\left|\frac{\theta_{4}}{\eta}\right|^{8}+\left|\frac{\theta_{2}}{\eta}\right|^{8}\right\}\left|\frac{\theta_{2}}{\eta}\right|^{8} .
\end{aligned}
$$

- $w \in 4 \mathbb{Z}+2$;

$$
\begin{aligned}
Z_{w}^{(\mathrm{NS}, \mathrm{NS})}(\tau, \bar{\tau})= & -Z_{w}^{(\mathrm{R}, \mathrm{NS})}(\tau, \bar{\tau})=\frac{1}{4} \sum_{n \in \mathbb{Z}+\frac{1}{2}} \overline{q^{\frac{1}{4}\left(\frac{n}{2 R}-R w\right)^{2}}} q^{\frac{1}{4}\left(\frac{n}{2 R}+R w\right)^{2}} \\
& \times\left\{\left|\frac{\theta_{3}}{\eta}\right|^{8}+\left|\frac{\theta_{4}}{\eta}\right|^{8}+\left|\frac{\theta_{2}}{\eta}\right|^{8}\right\} \overline{\left\{\left(\frac{\theta_{3}}{\eta}\right)^{4}+\left(\frac{\theta_{4}}{\eta}\right)^{4}\right\}}\left\{\left(\frac{\theta_{3}}{\eta}\right)^{4}-\left(\frac{\theta_{4}}{\eta}\right)^{4}\right\} \\
Z_{w}^{(\mathrm{R}, \mathrm{R})}(\tau, \bar{\tau})= & -Z_{w}^{(\mathrm{NS}, \mathrm{R})}(\tau, \bar{\tau})=\frac{1}{4} \sum_{n \in \mathbb{Z}} \overline{q^{\frac{1}{4}\left(\frac{n}{2 R}-R w\right)^{2}}} q^{\frac{1}{4}\left(\frac{n}{2 R}+R w\right)^{2}} \\
& \times\left\{\left|\frac{\theta_{3}}{\eta}\right|^{8}+\left|\frac{\theta_{4}}{\eta}\right|^{8}+\left|\frac{\theta_{2}}{\eta}\right|^{8}\right\}\left|\frac{\theta_{2}}{\eta}\right|^{8}
\end{aligned}
$$


- $w \in 2 \mathbb{Z}+1$

$$
\begin{aligned}
Z_{w}^{(\mathrm{NS}, \mathrm{NS})}(\tau, \bar{\tau})= & -Z_{w}^{(\mathrm{NS}, \mathrm{R})}(\tau, \bar{\tau})=\frac{1}{4} \sum_{r \in \mathbb{Z}_{2}} \sum_{n \in \mathbb{Z}} \overline{q^{\frac{1}{4}\left(\frac{n}{2 R}-R w\right)^{2}}} q^{\frac{1}{4}}\left(\frac{n}{2 R}+R w\right)^{2} \\
& \times(-1)^{r n} \overline{\left(\frac{\theta_{2} \theta_{3}\left(\frac{r}{2}\right)}{\eta^{2}}\right)^{4}}\left\{(-1)^{r}\left(\frac{\theta_{3}\left(\frac{r}{2}\right)}{\eta}\right)^{4}+\left(\frac{\theta_{2}}{\eta}\right)^{4}\right\}\left\{\left(\frac{\theta_{3}}{\eta}\right)^{4}+\left(\frac{\theta_{4}}{\eta}\right)^{4}\right\}, \\
Z_{w}^{(\mathrm{R}, \mathrm{R})}(\tau, \bar{\tau})= & -Z_{w}^{(\mathrm{R}, \mathrm{NS})}(\tau, \bar{\tau})=\frac{1}{4} \sum_{r \in \mathbb{Z}_{2}} \sum_{n \in \mathbb{Z}} \overline{q^{\frac{1}{4}\left(\frac{n}{2 R}-R w\right)^{2}}} q^{\frac{1}{4}\left(\frac{n}{2 R}+R w\right)^{2}} \\
& \times(-1)^{r n} \overline{\left(\frac{\theta_{2} \theta_{3}\left(\frac{r}{2}\right)}{\eta^{2}}\right)^{4}}\left\{\left(\frac{\theta_{3}\left(\frac{r}{2}\right)}{\eta}\right)^{4}+(-1)^{r}\left(\frac{\theta_{2}}{\eta}\right)^{4}\right\}\left(\frac{\theta_{2}}{\eta}\right)^{4} .
\end{aligned}
$$

Here, we denoted $\theta_{i} \equiv \theta_{i}(\tau, 0)$, and $\theta_{3}\left(\frac{r}{2}\right) \equiv \theta_{3}\left(\tau, \frac{r}{2}\right)$, and made use of the identity $\theta_{3}^{4}-\theta_{4}^{4}-\theta_{2}^{4}=0$

As expected, all of these partition functions are suitably $q$-expanded so as to be consistent with the unitarity.

The SUSY T-fold vacua (3.6), (3.7) are similarly analyzed. For the 3/4-SUSY vacuum (3.6), $Z_{w}^{(s, \tilde{s})}(\tau, \bar{\tau})$ becomes as follows:

- $w \in 2 \mathbb{Z}$

$$
Z_{w}^{(\mathrm{NS}, \mathrm{NS})}(\tau, \bar{\tau})=Z_{w}^{(\mathrm{R}, \mathrm{R})}(\tau, \bar{\tau})=-Z_{w}^{(\mathrm{R}, \mathrm{NS})}(\tau, \bar{\tau})=-Z_{w}^{(\mathrm{NS}, \mathrm{R})}(\tau, \bar{\tau})=(4.2)
$$

- $w \in 2 \mathbb{Z}+1$

$$
Z_{w}^{(\mathrm{NS}, \mathrm{NS})}(\tau, \bar{\tau})=Z_{w}^{(\mathrm{R}, \mathrm{R})}(\tau, \bar{\tau})=-Z_{w}^{(\mathrm{R}, \mathrm{NS})}(\tau, \bar{\tau})=-Z_{w}^{(\mathrm{NS}, \mathrm{R})}(\tau, \bar{\tau})=(4.7)
$$

On the other hand, for the 1/2-SUSY vacuum (3.7), we find the following:

- $w \in 4 \mathbb{Z}$

$$
\begin{aligned}
Z_{w}^{(\mathrm{NS}, \mathrm{NS})}(\tau, \bar{\tau}) & =-Z_{w}^{(\mathrm{R}, \mathrm{NS})}(\tau, \bar{\tau})=(4.2) \\
Z_{w}^{(\mathrm{R}, \mathrm{R})}(\tau, \bar{\tau}) & =-Z_{w}^{(\mathrm{NS}, \mathrm{R})}(\tau, \bar{\tau})=(4.3)
\end{aligned}
$$

- $w \in 4 \mathbb{Z}+2$;

$$
\begin{aligned}
Z_{w}^{(\mathrm{NS}, \mathrm{NS})}(\tau, \bar{\tau}) & =-Z_{w}^{(\mathrm{R}, \mathrm{NS})}(\tau, \bar{\tau})=(4.5) \\
Z_{w}^{(\mathrm{R}, \mathrm{R})}(\tau, \bar{\tau}) & =-Z_{w}^{(\mathrm{NS}, \mathrm{R})}(\tau, \bar{\tau})=(4.5)
\end{aligned}
$$

- $w \in 2 \mathbb{Z}+1$

$$
Z_{w}^{(\mathrm{NS}, \mathrm{NS})}(\tau, \bar{\tau})=Z_{w}^{(\mathrm{R}, \mathrm{R})}(\tau, \bar{\tau})=-Z_{w}^{(\mathrm{R}, \mathrm{NS})}(\tau, \bar{\tau})=-Z_{w}^{(\mathrm{NS}, \mathrm{R})}(\tau, \bar{\tau})=(4.7) .
$$


These analyses can be extended to other vacua built from $F_{(*, *)}^{T^{2}[\mathrm{SO}(4)] \times T^{2}[\mathrm{SO}(4)]}$ in (B.2) or $\widetilde{F}_{(*, *)}^{T^{2}[\mathrm{SO}(4)] \times T^{2}[\mathrm{SO}(4)]}$ in (B.4). In each case, we obtain the unitary $q$-expansion in a parallel way as above.

We remark that the above results (4.8) and (4.9) suggest that there are supercharges both from the left and right movers for the SUSY T-fold (3.6). Similarly, (4.10), (4.11) and (4.12) are consistent with the existence of the chiral SUSY that originates only from the left-mover. Then, how about the non-SUSY vacuum (3.9)? We note that, for instance,

$$
\begin{array}{ll}
Z_{w}^{(\mathrm{NS}, \mathrm{NS})}(\tau, \bar{\tau})=-Z_{w}^{(\mathrm{R}, \mathrm{NS})}(\tau, \bar{\tau}), & Z_{w}^{(\mathrm{R}, \mathrm{R})}(\tau, \bar{\tau})=-Z_{w}^{(\mathrm{NS}, \mathrm{R})}(\tau, \bar{\tau}), \\
Z_{w}^{(\mathrm{NS}, \mathrm{NS})}(\tau, \bar{\tau}) \neq-Z_{w}^{(\mathrm{NS}, \mathrm{R})}(\tau, \bar{\tau}), & Z_{w}^{(\mathrm{R}, \mathrm{R})}(\tau, \bar{\tau}) \neq-Z_{w}^{(\mathrm{R}, \mathrm{NS})}(\tau, \bar{\tau}),
\end{array}
$$

for $w \in 2 \mathbb{Z}$. These relations of the bose-fermi cancellation look as if we had left-moving SUSY, in spite that no supercharges exist in the left-mover in fact. On the other hand, we find

$$
\begin{array}{ll}
Z_{w}^{(\mathrm{NS}, \mathrm{NS})}(\tau, \bar{\tau})=-Z_{w}^{(\mathrm{NS}, \mathrm{R})}(\tau, \bar{\tau}), & Z_{w}^{(\mathrm{R}, \mathrm{R})}(\tau, \bar{\tau})=-Z_{w}^{(\mathrm{R}, \mathrm{NS})}(\tau, \bar{\tau}), \\
Z_{w}^{(\mathrm{NS}, \mathrm{NS})}(\tau, \bar{\tau}) \neq-Z_{w}^{(\mathrm{R}, \mathrm{NS})}(\tau, \bar{\tau}), & Z_{w}^{(\mathrm{R}, \mathrm{R})}(\tau, \bar{\tau}) \neq-Z_{w}^{(\mathrm{NS}, \mathrm{R})}(\tau, \bar{\tau}),
\end{array}
$$

for $w \in 2 \mathbb{Z}+1$, which would appear to be consistent with right-moving SUSY. We emphasize that any supercharges can never be compatible with both (4.13) and (4.14) at the same time. It may be an interesting issue whether such a curious feature is common to the vacua showing the bose-fermi cancellation without SUSY.

We also point out that the bose-fermi cancellation in (3.10) among different winding sectors does not happen (for arbitrary $w^{\prime}$ ), as is clear from the explicit forms of the partition functions presented above. Even at a special radius, the cancellation for arbitrary winding in (3.10) is not possible.

\subsection{Absence of winding tachyons}

Recall that our non-SUSY string vacuum (3.9) from $T^{4}[\mathrm{SO}(8)]$ has been constructed by including the Sherk-Schwarz type modification. Therefore, we would potentially face an issue of the instability caused by the winding tachyons that are typical in the SherkSchwarz compactification. That would be implied by the 'wrong GSO projections' observed in (4.5), (4.7). ${ }^{12}$ However, the spectrum is in fact free from the winding tachyons.

To show this, we first note that potentially dangerous states come from the winding sectors with $w \in 4 \mathbb{Z}+2$ or $w \in 2 \mathbb{Z}+1$, which are anticipated from the wrong GSO projections. Among them, we further focus on the NS-NS sector, since the spectrum is lifted in the R-R sector due to the $\theta_{2}$-factors, and the partition functions in the NS-R and R-NS sectors are the same as for the NS-NS or the R-R sector up to sign. From the partition functions (4.5), (4.7), we then find the following:

\footnotetext{
${ }^{12}$ In the T-fold vacuum (3.7), despite the existence of the space-time SUSY, we still find the wrong GSO fermions in the right-mover (with no SUSY), since $Z_{w}^{(\mathrm{NS}, \mathrm{NS})}\left(=-Z_{w}^{(\mathrm{R}, \mathrm{NS})}\right)$ coincides with the partition function (4.5) for $w \in 4 \mathbb{Z}+2$. Of course, one can confirm the absence of tachyonic modes in this model by a similar argument given here.
} 
- For $w \in 4 \mathbb{Z}+2$, the wrong GSO states are in the right-mover. The lightest excitations appear in the sectors of $w= \pm 2$, the conformal weights of which read

$$
h_{L}=\frac{1}{2}+\frac{1}{4}\left(\frac{n}{2 R} \pm 2 R\right)^{2}, \quad h_{R}=\frac{1}{4}\left(\frac{n}{2 R} \mp 2 R\right)^{2},
$$

with the KK momenta $n \in \mathbb{Z}+\frac{1}{2}$. Their minima for the physical states are achieved by setting $n=\mp \frac{1}{2}$, to give

$$
h_{L}=h_{R}=\frac{1}{2}+\frac{1}{4}\left(\frac{1}{4 R}-2 R\right)^{2} \geq \frac{1}{2} .
$$

This means that the winding states from these sectors are always massive except at the special radius $R=\frac{1}{2 \sqrt{2}}$ of the base circle, where extra massless excitations appear.

- For $w \in 2 \mathbb{Z}+1$, the wrong GSO states are in the left-mover. The lightest excitations appear in the sectors of $w= \pm 1$, and the leading contribution from the $\theta$-part comes from $\theta_{3}\left(\frac{r}{2}\right)=1+(-1)^{r} q^{\frac{1}{2}}+\cdots$. The summation over $r \in \mathbb{Z}_{2}$ then projects the KK momenta onto $n \in 2 \mathbb{Z}+1$, and the conformal weights read

$$
h_{L}=\frac{1}{4}\left(\frac{n}{2 R} \pm R\right)^{2}, \quad h_{R}=\frac{1}{2}+\frac{1}{4}\left(\frac{n}{2 R} \mp R\right)^{2}, \quad(n \in 2 \mathbb{Z}+1) .
$$

Their minima for the physical states are achieved by setting $n= \pm 1$, to give

$$
h_{L}=h_{R}=\frac{1}{2}+\frac{1}{4}\left(\frac{1}{2 R}-R\right)^{2} \geq \frac{1}{2} .
$$

This means that the winding states from these sectors are always massive except at the special radius $R=\frac{1}{\sqrt{2}}$, where extra massless excitations appear.

These demonstrate that no winding tachyons emerge in the non-SUSY vacuum (3.9).

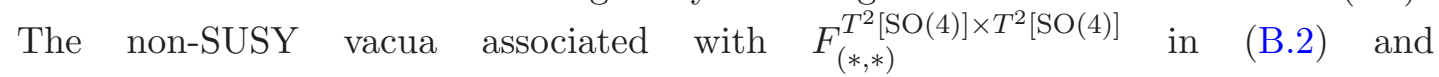
$\widetilde{F}_{(*, *)}^{T^{2}[\mathrm{SO}(4)] \times T^{2}[\mathrm{SO}(4)]}$ in (B.4) can be examined in a parallel way, and we obtain almost the same spectra of the winding excitations. However, there is a slight difference

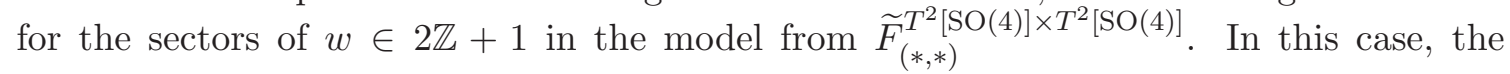
conformal weights of the $w= \pm 1$ sectors become

$$
h_{L}=\frac{1}{4}+\frac{1}{4}\left(\frac{n}{2 R} \pm R\right)^{2}, \quad h_{R}=\frac{1}{2}+\frac{1}{4}\left(\frac{n}{2 R} \mp R\right)^{2}, \quad\left(n \in \mathbb{Z}+\frac{1}{2}\right) .
$$

Here, $h_{L}$ also acquires the twisted energy from the extra $\theta_{2}$-factor. The KK momenta are shifted by one half due to the absence of the phase factors depending on the temporal winding $m$ (see (B.4)). Consequently, the lightest excitations lie in the sectors with $w=$ $\pm 1, n= \pm \frac{1}{2}$, giving

$$
h_{L}=h_{R}=\frac{1}{2}+\frac{1}{4}\left(\frac{1}{4 R}-R\right)^{2} \geq \frac{1}{2} .
$$

Again these are always massive except at the massless point $R=\frac{1}{2}$. 


\section{Summary and discussions}

In this paper, we have studied type II string vacua which are defined by the asymmetric orbifolding based on the chiral reflections/T-duality twists in $T^{4}$ combined with the shift in the base circle, in such a way that the modular invariance is kept manifest. They represent the non-geometric string vacua for T-folds, supposed that the world-sheet description of T-folds is generally given by asymmetric/generalized orbifolds. Including appropriate phases as in (B.4), the full OPE also respects the invariance under the T-duality twists in accord with [10]. As the main result, we have presented simple examples of the non-SUSY vacua with vanishing cosmological constant at one loop. We summarize the points to be emphasized as follows:

- Our non-SUSY vacuum (3.9) has been defined by a cyclic orbifold which is generated by a single element $g$ in (3.8). Thus, it provides a simpler model than the previous ones [21-27]. In this construction, taking both the asymmetric orbifold action with $\sigma^{2}=(-1)^{F_{R}}$ and the Scherk-Schwarz compactification (orbifolding by $\left.\left.(-1)^{F_{L}} \otimes \mathcal{T}_{2 \pi R}\right|_{\text {base }}\right)$ at the same time is truly crucial in order to make the SUSYbreaking compatible with the bose-fermi cancellation. Indeed, it is important that the left and right-moving non-SUSY chiral blocks $\mathbf{f}_{(*, *)}(\tau), \overline{\mathbf{f}_{(*, *)}(\tau)}$, which originate from the SUSY-breaking twists $(-1)^{F_{L}},(-1)^{F_{R}}$, depend on the winding numbers along the Scherk-Schwarz circle in an asymmetric way.

- The modular invariant partition function given in (3.9) is $q$-expanded so as to be compatible with unitarity, as shown in subsection 4.2. Curiously, it turns out that the left-moving bose-fermi cancellation occurs in the even winding sectors, while we have the right-moving bose-fermi cancellation in the odd winding sectors. This aspect is in sharp contrast with any SUSY vacua.

- Despite the absence of the space-time SUSY and adopting the Scherk-Schwarz type compactification, we are free from the tachyonic instability at any radius of the Scherk-Schwarz circle.

To conclude, we would like to make a few comments on possible future studies. First of all, it would indeed be an interesting issue whether our non-SUSY vacuum (3.9) has vanishing cosmological constant at higher loops. Since the orbifold structure of this vacuum is simpler than those of the previous ones quoted above, it would be worthwhile to examine especially the two-loop case by following the analysis in [28].

Secondly, in order to search a more broad class of such vacua, one may extend the construction in this paper to other toroidal models of asymmetric orbifolds. Furthermore, toward more realistic models, it would also be important to consider the non-geometric string vacua from SCFTs other than the toroidal ones. For previous attempts based on the $\mathcal{N}=2$ SCFTs, see e.g. [12]. A challenging direction in this respect, and along [15], would be to construct such vacua based on the generalized orbifolds through the topological interfaces, which are wrapped around the cycles of the world-sheet torus in correlation 
with the shift operators. ${ }^{13}$ The point here would be how to organize the world-sheet chiral sectors depending on the winding numbers along the Scherk-Schwarz like circle, so that the bose-fermi cancellation does occur. We expect that the novel feature of the cancellation, which is remarked at the end of subsection 4.2 , would be observed only in the non-geometric backgrounds.

Thirdly, one may also extend this work so as to include the open string sectors, namely, D-branes. Possibilities of the bose-fermi cancellation in the open string Hilbert space have been investigated [36] under particular SUSY breaking configurations of D-branes. Closely related studies of D-branes in asymmetric orbifolds by the T-duality twists have been presented e.g. in [11, 48-50]. It would be interesting to study the aspects of D-branes in the type II vacua given in this paper (and their variants), in comparison with these previous works.

\section{Acknowledgments}

We would like to thank K. Aoki for a useful conversation. This work is supported in part by JSPS KAKENHI Grant Number 24540248 and 23540322 from Japan Society for the Promotion of Science (JSPS).

\section{A Summary of conventions and useful formulas}

\section{Theta functions:}

$$
\begin{aligned}
\theta_{1}(\tau, z) & :=i \sum_{n=-\infty}^{\infty}(-1)^{n} q^{(n-1 / 2)^{2} / 2} y^{n-1 / 2} \equiv 2 \sin (\pi z) q^{1 / 8} \prod_{m=1}^{\infty}\left(1-q^{m}\right)\left(1-y q^{m}\right)\left(1-y^{-1} q^{m}\right), \\
\theta_{2}(\tau, z) & :=\sum_{n=-\infty}^{\infty} q^{(n-1 / 2)^{2} / 2} y^{n-1 / 2} \equiv 2 \cos (\pi z) q^{1 / 8} \prod_{m=1}^{\infty}\left(1-q^{m}\right)\left(1+y q^{m}\right)\left(1+y^{-1} q^{m}\right), \\
\theta_{3}(\tau, z) & :=\sum_{n=-\infty}^{\infty} q^{n^{2} / 2} y^{n} \equiv \prod_{m=1}^{\infty}\left(1-q^{m}\right)\left(1+y q^{m-1 / 2}\right)\left(1+y^{-1} q^{m-1 / 2}\right), \\
\theta_{4}(\tau, z) & :=\sum_{n=-\infty}^{\infty}(-1)^{n} q^{n^{2} / 2} y^{n} \equiv \prod_{m=1}^{\infty}\left(1-q^{m}\right)\left(1-y q^{m-1 / 2}\right)\left(1-y^{-1} q^{m-1 / 2}\right) . \\
\Theta_{m, k}(\tau, z) & :=\sum_{n=-\infty}^{\infty} q^{k\left(n+\frac{m}{2 k}\right)^{2}} y^{k\left(n+\frac{m}{2 k}\right)}, \\
\widetilde{\Theta}_{m, k}(\tau, z) & :=\sum_{n=-\infty}^{\infty}(-1)^{n} q^{k\left(n+\frac{m}{2 k}\right)^{2}} y^{k\left(n+\frac{m}{2 k}\right)}, \\
\eta(\tau) & :=q^{1 / 24} \prod_{n=1}^{\infty}\left(1-q^{n}\right) .
\end{aligned}
$$

\footnotetext{
${ }^{13}$ For applications of the world-sheet conformal interfaces to string theory, see e.g. [44-47].
} 
Here, we have set $q:=e^{2 \pi i \tau}, y:=e^{2 \pi i z}\left({ }^{\forall} \tau \in \mathbb{H}^{+},{ }^{\forall} z \in \mathbb{C}\right)$, and used abbreviations, $\theta_{i}(\tau) \equiv \theta_{i}(\tau, 0)\left(\theta_{1}(\tau) \equiv 0\right), \Theta_{m, k}(\tau) \equiv \Theta_{m, k}(\tau, 0)$. It is straightforward to prove the following identities:

$$
\frac{\widetilde{\Theta}_{0,1}(\tau)}{\eta(\tau)}=\sqrt{\frac{2 \eta(\tau)}{\theta_{2}(\tau)}}, \quad \frac{\Theta_{1 / 2,1}(\tau)}{\eta(\tau)}=\sqrt{\frac{\eta(\tau)}{\theta_{4}(\tau)}}, \quad \frac{\widetilde{\Theta}_{1 / 2,1}(\tau)}{\eta(\tau)}=\sqrt{\frac{\eta(\tau)}{\theta_{3}(\tau)}} .
$$

\section{Poisson resummation formula:}

$$
\begin{gathered}
\sum_{n \in \mathbb{Z}} \exp \left(-\pi \alpha(n+a)^{2}+2 \pi i b(n+a)\right)=\frac{1}{\sqrt{\alpha}} \sum_{m \in \mathbb{Z}} \exp \left(-\frac{\pi(m-b)^{2}}{\alpha}+2 \pi i m a\right), \\
(\alpha>0, a, b \in \mathbb{R}) .
\end{gathered}
$$

\section{B Summary of building blocks}

In appendix B, we summarize the notations of relevant building blocks to construct the torus partition functions used in the main text.

\section{Bulidng blocks for the bosonic $T^{4}$-secotor:}

\section{Chiral reflection in $T^{4}[\mathrm{SO}(8)]$ :}

$$
F_{(a, b)}^{T^{4}[\operatorname{SO}(8)]}(\tau, \bar{\tau})= \begin{cases}(-1)^{\frac{a}{2}} \overline{\left(\frac{\theta_{3} \theta_{4}}{\eta^{2}}\right)^{2}} \cdot \frac{1}{2}\left\{\left(\frac{\theta_{3}}{\eta}\right)^{4}+\left(\frac{\theta_{4}}{\eta}\right)^{4}\right\} & (a \in 2 \mathbb{Z}, b \in 2 \mathbb{Z}+1), \\ (-1)^{\frac{b}{2}} \overline{\left.\frac{\theta_{2} \theta_{3}}{\eta^{2}}\right)^{2}} \cdot \frac{1}{2}\left\{\left(\frac{\theta_{3}}{\eta}\right)^{4}+\left(\frac{\theta_{2}}{\eta}\right)^{4}\right\} & (a \in 2 \mathbb{Z}+1, b \in 2 \mathbb{Z}), \\ e^{-\frac{i \pi}{2} a b} \overline{\left(\frac{\theta_{4} \theta_{2}}{\eta^{2}}\right)^{2}} \cdot \frac{1}{2}\left\{\left(\frac{\theta_{4}}{\eta}\right)^{4}-\left(\frac{\theta_{2}}{\eta}\right)^{4}\right\} & (a \in 2 \mathbb{Z}+1, b \in 2 \mathbb{Z}+1), \\ \frac{1}{2}\left\{\left|\frac{\theta_{3}}{\eta}\right|^{8}+\left|\frac{\theta_{4}}{\eta}\right|^{8}+\left|\frac{\theta_{2}}{\eta}\right|^{8}\right\} & (a \in 2 \mathbb{Z}, b \in 2 \mathbb{Z}) .\end{cases}
$$

Of course, $F_{(a, b)}^{T^{4}[\mathrm{SO}(8)]}$ for the $a, b \in 2 \mathbb{Z}$ case coincides with the original partition function $Z_{T^{4}[\mathrm{SO}(8)]}(2.3)$.

2. Chiral reflection in $T^{2}[\mathrm{SO}(4)] \times T^{2}[\mathrm{SO}(4)]$ :

$$
\begin{aligned}
& F_{(a, b)}^{T^{2}[\mathrm{SO}(4)] \times T^{2}[\mathrm{SO}(4)](\tau, \bar{\tau})} \\
& = \begin{cases}(-1)^{\frac{a}{2}} \overline{\left(\frac{\theta_{3} \theta_{4}}{\eta^{2}}\right)^{2}} \cdot \frac{1}{4}\left\{\left(\frac{\theta_{3}}{\eta}\right)^{2}+(-1)^{\frac{a}{2}}\left(\frac{\theta_{4}}{\eta}\right)^{2}\right\}^{2} & (a \in 2 \mathbb{Z}, b \in 2 \mathbb{Z}+1), \\
(-1)^{\frac{b}{2}} \overline{\left.\frac{\theta_{2} \theta_{3}}{\eta^{2}}\right)^{2}} \cdot \frac{1}{4}\left\{\left(\frac{\theta_{3}}{\eta}\right)^{2}+(-1)^{\frac{b}{2}}\left(\frac{\theta_{2}}{\eta}\right)^{2}\right\}^{2} & (a \in 2 \mathbb{Z}+1, b \in 2 \mathbb{Z}), \\
e^{-\frac{i \pi}{2} a b} \overline{\left(\frac{\theta_{4} \theta_{2}}{\eta^{2}}\right)^{2}} \cdot \frac{1}{4}\left\{\left(\frac{\theta_{4}}{\eta}\right)^{2}-i(-1)^{\frac{a+b}{2}}\left(\frac{\theta_{2}}{\eta}\right)^{2}\right\}^{2} & (a \in 2 \mathbb{Z}+1, b \in 2 \mathbb{Z}+1), \\
\frac{1}{4}\left\{\left|\frac{\theta_{3}}{\eta}\right|^{4}+\left|\frac{\theta_{4}}{\eta}\right|^{4}+\left|\frac{\theta_{2}}{\eta}\right|^{4}\right\}^{2} & (a \in 2 \mathbb{Z}, b \in 2 \mathbb{Z}) .\end{cases}
\end{aligned}
$$


Here, $F_{(a, b)}^{T^{2}[\mathrm{SO}(4)] \times T^{2}[\mathrm{SO}(4)]}$ for $a, b \in 2 \mathbb{Z}$ coincides with the partition function $Z_{T^{2}[\mathrm{SO}(4)] \times T^{2}[\mathrm{SO}(4)]}(2.4)$, and we also find the identities

$$
\begin{aligned}
\frac{1}{4}\left\{\left|\frac{\theta_{3}}{\eta}\right|^{4}+\left|\frac{\theta_{4}}{\eta}\right|^{4}+\left|\frac{\theta_{2}}{\eta}\right|^{4}\right\}^{2} & =\frac{1}{2}\left[Z_{T^{4}[\mathrm{SO}(8)]}(\tau, \bar{\tau})+\left|\frac{\theta_{3} \theta_{4}}{\eta^{2}}\right|^{4}+\left|\frac{\theta_{4} \theta_{2}}{\eta^{2}}\right|^{4}+\left|\frac{\theta_{2} \theta_{3}}{\eta^{2}}\right|^{4}\right] \\
& =\left[\left|\frac{\Theta_{0,1}}{\eta}\right|^{2}+\left|\frac{\Theta_{1,1}}{\eta}\right|^{2}\right]^{4}
\end{aligned}
$$

They obviously show the equivalence (2.5).

3. Chiral reflection in $T^{2}[\mathrm{SO}(4)] \times T^{2}[\mathrm{SO}(4)]$ with a phase factor:

$$
\widetilde{F}_{(a, b)}^{T^{2}[\mathrm{SO}(4)] \times T^{2}[\mathrm{SO}(4)]}(\tau, \bar{\tau})= \begin{cases}\left|\frac{\theta_{3} \theta_{4}}{\eta^{2}}\right|^{4} & (a \in 2 \mathbb{Z}, b \in 2 \mathbb{Z}+1), \\ \left|\frac{\theta_{2} \theta_{3}}{\eta^{2}}\right|^{4} & (a \in 2 \mathbb{Z}+1, b \in 2 \mathbb{Z}), \\ \left|\frac{\theta_{4} \theta_{2}}{\eta^{2}}\right|^{4} & (a \in 2 \mathbb{Z}+1, b \in 2 \mathbb{Z}+1), \\ \frac{1}{4}\left\{\left|\frac{\theta_{3}}{\eta}\right|^{4}+\left|\frac{\theta_{4}}{\eta}\right|^{4}+\left|\frac{\theta_{2}}{\eta}\right|^{4}\right\}^{2} & (a \in 2 \mathbb{Z}, b \in 2 \mathbb{Z}) .\end{cases}
$$

Again the building block for $a, b \in 2 \mathbb{Z}$ coincides with (2.4), and the blocks for the $(0, b)$-sectors with $b \in 2 \mathbb{Z}+1$ are explicitly computed as

$$
\begin{aligned}
\widetilde{F}_{(0, b)}^{T^{2}[\mathrm{SO}(4)] \times T^{2}[\mathrm{SO}(4)]}(\tau, \bar{\tau}) & =\left[\sqrt{\sqrt{\frac{2 \eta}{\theta_{2}}}} \cdot \frac{1}{\eta} \sum_{n \in \mathbb{Z}}(-1)^{n} q^{n^{2}}\right]^{4} \\
& =\left|\frac{\theta_{3} \theta_{4}}{\eta^{2}}\right|^{4}
\end{aligned}
$$

Here, we used the identity (A.8) and the Euler identity $\theta_{2} \theta_{3} \theta_{4}=2 \eta^{3}$ to derive the second line. The building blocks of other sectors are obtained by requiring the modular covariance, and one can quickly reproduce the results (B.4).

\section{Chiral building blocks for the fermionic sector:}

(i) For the case $\sigma_{L}^{2}=1$ :

$$
\begin{aligned}
f_{(a, b)}(\tau)= & 2 q^{\frac{1}{4} a^{2}} e^{\frac{i \pi}{2} a b}\left(\frac{\theta_{1}\left(\tau, \frac{a \tau+b}{2}\right)}{\eta(\tau)}\right)^{2}\left(\frac{\theta_{1}(\tau, 0)}{\eta(\tau)}\right)^{2} \\
& \equiv \begin{cases}(-1)^{\frac{a}{2}}\left\{\left(\frac{\theta_{3}}{\eta}\right)^{2}\left(\frac{\theta_{4}}{\eta}\right)^{2}-\left(\frac{\theta_{4}}{\eta}\right)^{2}\left(\frac{\theta_{3}}{\eta}\right)^{2}+0\right\} & (a \in 2 \mathbb{Z}, b \in 2 \mathbb{Z}+1), \\
(-1)^{\frac{b}{2}}\left\{\left(\frac{\theta_{3}}{\eta}\right)^{2}\left(\frac{\theta_{2}}{\eta}\right)^{2}+0-\left(\frac{\theta_{2}}{\eta}\right)^{2}\left(\frac{\theta_{3}}{\eta}\right)^{2}\right\} & (a \in 2 \mathbb{Z}+1, b \in 2 \mathbb{Z}), \\
-e^{\frac{i \pi}{2} a b}\left\{0+\left(\frac{\theta_{2}}{\eta}\right)^{2}\left(\frac{\theta_{4}}{\eta}\right)^{2}-\left(\frac{\theta_{4}}{\eta}\right)^{2}\left(\frac{\theta_{2}}{\eta}\right)^{2}\right\} & (a \in 2 \mathbb{Z}+1, b \in 2 \mathbb{Z}+1), \\
\left(\frac{\theta_{3}}{\eta}\right)^{4}-\left(\frac{\theta_{4}}{\eta}\right)^{4}-\left(\frac{\theta_{2}}{\eta}\right)^{4} & (a \in 2 \mathbb{Z}, b \in 2 \mathbb{Z}) .\end{cases}
\end{aligned}
$$


Since $f_{(a, b)}(\tau)$ actually vanish, it is better to first consider

$$
f_{(a, b)}(\tau, \epsilon) \equiv 2 q^{\frac{1}{4} a^{2}} e^{\frac{i \pi}{2} a b}\left(\frac{\theta_{1}\left(\tau, \frac{a \tau+b}{2}\right)}{\eta(\tau)}\right)^{2}\left(\frac{\theta_{1}(\tau, \epsilon)}{\eta(\tau)}\right)^{2}
$$

in order to express the modular covariance relation with no subtlety, One should then interpret (2.24) as the $\epsilon \rightarrow 0$ limit of

$$
\begin{aligned}
\left.f_{a, b}(\tau, \epsilon)\right|_{S} & \equiv f_{(a, b)}\left(-\frac{1}{\tau}, \frac{\epsilon}{\tau}\right)=e^{i \pi \frac{2}{\tau} \epsilon^{2}} f_{(b,-a)}(\tau, \epsilon), \\
\left.f_{(a, b)}(\tau, \epsilon)\right|_{T} & \equiv f_{(a, b)}(\tau+1, \epsilon)=-e^{-2 \pi i \frac{1}{6}} f_{(a, a+b)}(\tau, \epsilon) .
\end{aligned}
$$

(ii) For the case of $\sigma_{L}^{2}=(-1)^{F_{L}}$ :

$$
\mathbf{f}_{(a, b)}(\tau)=f_{(a, b)}(\tau), \quad(a \in 2 \mathbb{Z}+1 \text { or } b \in 2 \mathbb{Z}+1),
$$

and

$$
\mathbf{f}_{(a, b)}(\tau)= \begin{cases}\left(\frac{\theta_{3}}{\eta}\right)^{4}-\left(\frac{\theta_{4}}{\eta}\right)^{4}-\left(\frac{\theta_{2}}{\eta}\right)^{4} & (a \in 4 \mathbb{Z}, b \in 4 \mathbb{Z}), \\ \left(\frac{\theta_{3}}{\eta}\right)^{4}-\left(\frac{\theta_{4}}{\eta}\right)^{4}+\left(\frac{\theta_{2}}{\eta}\right)^{4} & (a \in 4 \mathbb{Z}, b \in 4 \mathbb{Z}+2), \\ \left(\frac{\theta_{3}}{\eta}\right)^{4}+\left(\frac{\theta_{4}}{\eta}\right)^{4}-\left(\frac{\theta_{2}}{\eta}\right)^{4} & (a \in 4 \mathbb{Z}+2, b \in 4 \mathbb{Z}), \\ -\left\{\left(\frac{\theta_{3}}{\eta}\right)^{4}+\left(\frac{\theta_{4}}{\eta}\right)^{4}+\left(\frac{\theta_{2}}{\eta}\right)^{4}\right\} & (a \in 4 \mathbb{Z}+2, b \in 4 \mathbb{Z}+2) .\end{cases}
$$

Open Access. This article is distributed under the terms of the Creative Commons Attribution License (CC-BY 4.0), which permits any use, distribution and reproduction in any medium, provided the original author(s) and source are credited.

\section{References}

[1] A. Dabholkar and C. Hull, Duality twists, orbifolds and fluxes, JHEP 09 (2003) 054 [hep-th/0210209] [INSPIRE].

[2] S. Hellerman, J. McGreevy and B. Williams, Geometric constructions of nongeometric string theories, JHEP 01 (2004) 024 [hep-th/0208174] [INSPIRE].

[3] A. Flournoy, B. Wecht and B. Williams, Constructing nongeometric vacua in string theory, Nucl. Phys. B 706 (2005) 127 [hep-th/0404217] [INSPIRE].

[4] C.M. Hull, A Geometry for non-geometric string backgrounds, JHEP 10 (2005) 065 [hep-th/0406102] [INSPIRE].

[5] J. Shelton, W. Taylor and B. Wecht, Nongeometric flux compactifications, JHEP 10 (2005) 085 [hep-th/0508133] [INSPIRE].

[6] J. Shelton, W. Taylor and B. Wecht, Generalized Flux Vacua, JHEP 02 (2007) 095 [hep-th/0607015] [INSPIRE]. 
[7] A. Dabholkar and C. Hull, Generalised T-duality and non-geometric backgrounds, JHEP 05 (2006) 009 [hep-th/0512005] [INSPIRE].

[8] K.S. Narain, M.H. Sarmadi and C. Vafa, Asymmetric Orbifolds, Nucl. Phys. B 288 (1987) 551 [inSPIRE].

[9] A. Flournoy and B. Williams, Nongeometry, duality twists and the worldsheet, JHEP 01 (2006) 166 [hep-th/0511126] [INSPIRE].

[10] S. Hellerman and J. Walcher, Worldsheet CFTs for Flat Monodrofolds, hep-th/0604191 [INSPIRE].

[11] S. Kawai and Y. Sugawara, D-branes in T-fold conformal field theory, JHEP 02 (2008) 027 [arXiv: 0709.0257] [INSPIRE].

[12] S. Kawai and Y. Sugawara, Mirrorfolds with K3 fibrations, JHEP 02 (2008) 065 [arXiv:0711.1045] [INSPIRE].

[13] C. Condeescu, I. Florakis and D. Lüst, Asymmetric Orbifolds, Non-Geometric Fluxes and Non-Commutativity in Closed String Theory, JHEP 04 (2012) 121 [arXiv:1202.6366] [INSPIRE].

[14] C. Condeescu, I. Florakis, C. Kounnas and D. Lüst, Gauged supergravities and non-geometric Q/R-fluxes from asymmetric orbifold CFT's, JHEP 10 (2013) 057 [arXiv:1307.0999] [INSPIRE].

[15] Y. Satoh and Y. Sugawara, Non-geometric Backgrounds Based on Topological Interfaces, JHEP 07 (2015) 022 [arXiv: 1502.05776] [INSPIRE].

[16] H.S. Tan, T-duality Twists and Asymmetric Orbifolds, JHEP 11 (2015) 141 [arXiv: 1508.04807] [INSPIRE].

[17] R. Blumenhagen and E. Plauschinn, Nonassociative Gravity in String Theory?, J. Phys. A 44 (2011) 015401 [arXiv:1010.1263] [INSPIRE].

[18] D. Lüst, T-duality and closed string non-commutative (doubled) geometry, JHEP 12 (2010) 084 [arXiv:1010.1361] [InSPIRE].

[19] R. Blumenhagen, A. Deser, D. Lüst, E. Plauschinn and F. Rennecke, Non-geometric Fluxes, Asymmetric Strings and Nonassociative Geometry, J. Phys. A 44 (2011) 385401 [arXiv:1106.0316] [INSPIRE].

[20] D. Andriot, M. Larfors, D. Lüst and P. Patalong, (Non-)commutative closed string on T-dual toroidal backgrounds, JHEP 06 (2013) 021 [arXiv:1211.6437] [INSPIRE].

[21] S. Kachru, J. Kumar and E. Silverstein, Vacuum energy cancellation in a nonsupersymmetric string, Phys. Rev. D 59 (1999) 106004 [hep-th/9807076] [INSPIRE].

[22] S. Kachru and E. Silverstein, Selfdual nonsupersymmetric type-II string compactifications, JHEP 11 (1998) 001 [hep-th/9808056] [INSPIRE].

[23] S. Kachru and E. Silverstein, On vanishing two loop cosmological constants in nonsupersymmetric strings, JHEP 01 (1999) 004 [hep-th/9810129] [INSPIRE].

[24] J.A. Harvey, String duality and nonsupersymmetric strings, Phys. Rev. D 59 (1999) 026002 [hep-th/9807213] [INSPIRE].

[25] G. Shiu and S.H.H. Tye, Bose-Fermi degeneracy and duality in nonsupersymmetric strings, Nucl. Phys. B 542 (1999) 45 [hep-th/9808095] [INSPIRE]. 
[26] R. Blumenhagen and L. Görlich, Orientifolds of nonsupersymmetric asymmetric orbifolds, Nucl. Phys. B 551 (1999) 601 [hep-th/9812158] [INSPIRE].

[27] C. Angelantonj, I. Antoniadis and K. Forger, Nonsupersymmetric type-I strings with zero vacuum energy, Nucl. Phys. B 555 (1999) 116 [hep-th/9904092] [INSPIRE].

[28] K. Aoki, E. D'Hoker and D.H. Phong, Two loop superstrings on orbifold compactifications, Nucl. Phys. B 688 (2004) 3 [hep-th/0312181] [INSPIRE].

[29] M. Blaszczyk, S. Groot Nibbelink, O. Loukas and S. Ramos-Sanchez, Non-supersymmetric heterotic model building, JHEP 10 (2014) 119 [arXiv:1407.6362] [INSPIRE].

[30] C. Angelantonj, I. Florakis and M. Tsulaia, Phys. Lett. B 736 (2014) 365 [arXiv: 1407.8023] [INSPIRE].

[31] A.E. Faraggi, C. Kounnas and H. Partouche, Large volume SUSY breaking with a solution to the decompactification problem, Nucl. Phys. B 899 (2015) 328 [arXiv:1410.6147] [INSPIRE].

[32] S. Abel, K.R. Dienes and E. Mavroudi, Towards a nonsupersymmetric string phenomenology, Phys. Rev. D 91 (2015) 126014 [arXiv: 1502.03087] [INSPIRE].

[33] J. Scherk and J.H. Schwarz, Spontaneous Breaking of Supersymmetry Through Dimensional Reduction, Phys. Lett. B 82 (1979) 60 [INSPIRE].

[34] J. Scherk and J.H. Schwarz, How to Get Masses from Extra Dimensions, Nucl. Phys. B 153 (1979) 61 [inSPIRE].

[35] K. Aoki, E. D'Hoker and D.H. Phong, On the construction of asymmetric orbifold models, Nucl. Phys. B 695 (2004) 132 [hep-th/0402134] [INSPIRE].

[36] M.R. Gaberdiel and A. Sen, Nonsupersymmetric D-brane configurations with Bose-Fermi degenerate open string spectrum, JHEP 11 (1999) 008 [hep-th/9908060] [INSPIRE].

[37] J. Fröhlich, J. Fuchs, I. Runkel and C. Schweigert, Defect lines, dualities and generalised orbifolds, arXiv:0909.5013 [INSPIRE].

[38] N. Carqueville and I. Runkel, Orbifold completion of defect bicategories, arXiv:1210.6363 [INSPIRE].

[39] I. Brunner, N. Carqueville and D. Plencner, Orbifolds and topological defects, Commun. Math. Phys. 332 (2014) 669 [arXiv:1307.3141] [InSPIRE].

[40] E. Wong and I. Affleck, Tunneling in quantum wires: A Boundary conformal field theory approach, Nucl. Phys. B 417 (1994) 403 [inSPIRE].

[41] V.B. Petkova and J.B. Zuber, Generalized twisted partition functions, Phys. Lett. B 504 (2001) 157 [hep-th/0011021] [INSPIRE].

[42] C. Bachas, J. de Boer, R. Dijkgraaf and H. Ooguri, Permeable conformal walls and holography, JHEP 06 (2002) 027 [hep-th/0111210] [INSPIRE].

[43] C. Bachas and M. Gaberdiel, Loop operators and the Kondo problem, JHEP 11 (2004) 065 [hep-th/0411067] [INSPIRE].

[44] C. Bachas and I. Brunner, Fusion of conformal interfaces, JHEP 02 (2008) 085 [arXiv:0712.0076] [INSPIRE].

[45] Y. Satoh, On supersymmetric interfaces for string theory, JHEP 03 (2012) 072 [arXiv:1112.5935] [INSPIRE]. 
[46] C. Bachas, I. Brunner and D. Roggenkamp, A worldsheet extension of $O(d, d: Z)$, JHEP 10 (2012) 039 [arXiv: 1205.4647] [INSPIRE].

[47] S. Elitzur, B. Karni, E. Rabinovici and G. Sarkissian, Defects, Super-Poincaré line bundle and Fermionic T-duality, JHEP 04 (2013) 088 [arXiv: 1301.6639] [INSPIRE].

[48] I. Brunner, A. Rajaraman and M. Rozali, D-branes on asymmetric orbifolds, Nucl. Phys. B 558 (1999) 205 [hep-th/9905024] [INSPIRE].

[49] M.R. Gaberdiel and S. Schäfer-Nameki, D-branes in an asymmetric orbifold, Nucl. Phys. B 654 (2003) 177 [hep-th/0210137] [INSPIRE].

[50] M. Bianchi, Bound-states of D-branes in L-R asymmetric superstring vacua, Nucl. Phys. B 805 (2008) 168 [arXiv:0805.3276] [INSPIRE]. 